\title{
TRANSFERRING OPTIMAL DUALITIES: THEORY AND PRACTICE
}

\author{
B. A. DAVEY and M. HAVIAR \\ Dedicated to the memory of Dorothy Berridge
}

(Received 22 May 2000; revised 22 April 2002)

Communicated by C. F. Miller

\begin{abstract}
Consider the quasi-variety $\mathcal{D}$ generated by a finite algebra $\underline{\mathbf{D}}$ and assume that $\underline{\mathbf{D}}$ yields a natural duality on $\mathcal{D}$ based on $\underline{\mathbf{D}}$ which is optimal modulo endomorphisms. We show that, provided $\mathbf{D}$ satisfies certain minimality conditions, we can transfer this duality to a natural duality on $\mathcal{D}$ based on $\underline{\mathbf{M}}$, which is also optimal modulo endomorphisms, for any finite algebra $\underline{\mathbf{M}}$ in $\mathcal{D}$ that has a subalgebra isomorphic to $\underline{\mathbf{D}}$.

2000 Mathematics subject classification: primary 08C15, 08A99, 06D50, 06D15, 06D30.

Keywords and phrases: natural duality, optimal duality, endodualisability, endoprimality, globally minimal failset, entailment, retraction, distributive lattice, Kleene algebra, Stone algebra.
\end{abstract}

\section{Introduction and preliminaries}

The theory of natural dualities deals with the topological representation of algebras. The main idea is that, given a quasi-variety $\mathcal{D}:=\mathbb{\triangle S} \mathbb{P}(\underline{D})$ generated by a finite algebra $\underline{\mathbf{D}}=\langle D ; F\rangle$, we seek a topological structure $\mathbf{\mathbf { D }}=\langle D ; G, H, R, \mathscr{T}\rangle$ on the underlying set $D$ of $\underline{\mathbf{D}}$ such that a dual equivalence exists between $\mathcal{D}$ and a suitable category $X$ of topological structures of the same type as $\underset{\sim}{\mathbf{D}}$. (Here $G, H$ and $R$ are sets of finitary operations, partial operations and relations on $D$ and $\mathscr{T}$ is the discrete topology.) As a result, we obtain a uniform way of representing each algebra $\mathbf{A}$ in the quasi-variety $\mathcal{D}$

The support of a La Trobe University Postdoctoral Research Fellowship and Slovak VEGA grant $1 / 7490 / 20$ are acknowledged by the second author. The authors thank Jane Pitkethly for many helpful remarks.

(C) 2003 Australian Mathematical Society $1446-7887 / 03 \$ A 2.00+0.00$ 
as an algebra, $\boldsymbol{X}(\mathbf{X}, \underset{\sim}{\mathbf{D}})$, of continuous structure-preserving maps from a dual $\mathbf{X} \in \boldsymbol{X}$ of the algebra $\mathbf{A}$ into $\mathbf{D}$.

On the one hand, the theory of natural dualities strives to give us an insight into the general structure of the quasi-variety $\mathcal{D}:=\square \mathbb{S P}(\mathbb{D})$. At this level, we are asking what it is about the algebra $\underline{\mathbf{D}}$ and the quasi-variety $\mathcal{D}$ that will guarantee that there is some structure $\mathbf{D}=\langle D ; G, H, R, \mathscr{T}\rangle$ which yields a natural duality on $\mathcal{D}$. On the other hand, once we have a dualising structure $\underset{\sim}{\mathbf{D}}$, it is natural to ask how it may be refined, by deleting members of $G \cup H \cup R$, to obtain an optimal duality. The focus of the present paper is on the optimal-duality end of this spectrum. A number of authors, notably H.A. Priestley and her students, have considered optimal dualities: see Davey and Priestley [11-13], Davey, Haviar and Priestley [7,8], Saramago and Priestley [24], Saramago [22], Wegener [25] and Clark and Davey [2, Chapter 8].

A reader who is well versed in the basics of the theory of natural dualities may like to skip to the last four paragraphs of this section where we describe, in general terms, the main results and applications to be found in Sections 2 to 4 .

Let $\underline{\mathrm{D}}=\langle D ; G, H, R, \mathscr{T}\rangle$ be algebraic over $\underline{\mathbf{D}}$, meaning that all relations in $R$ and graphs of all (partial) operations in $G \cup H$ are algebraic on $\underline{\mathbf{D}}$, that is, are subalgebras of appropriate powers of $\underline{\mathbf{D}}$. Let $\boldsymbol{X}=\mathbb{\backslash} \mathbb{S}_{c} \mathbb{P}(\underline{\mathbf{D}})$ denote the class of all topological structures that are isomorphic to closed substructures of powers $\mathbf{D}^{S}$ for non-empty sets $S$. For $\mathbf{A} \in \mathcal{D}$, let $D(\mathbf{A}):=\mathcal{D}(\mathbf{A}, \underline{\mathbf{D}})$ be the homset consisting of all $\mathcal{D}$ homomorphisms from $\mathbf{A}$ to $\underline{\mathbf{D}}$. A relation $r \subseteq D^{n}$ induces a relation $r^{D(\mathbf{A})}$ on the dual $D(\mathbf{A})$ as follows: for $x_{1}, \ldots, x_{n} \in D(\mathbf{A})$ we write $\left(x_{1}, \ldots, x_{n}\right) \in r^{D(\mathbf{A})}$ if and only if $\left(x_{1}(a), \ldots, x_{n}(a)\right) \in r$ for all $a \in A$. We say that a map $\alpha: D(\mathbf{A}) \rightarrow D$ preserves the relation $r$ if $\left(\alpha\left(x_{1}\right), \ldots, \alpha\left(x_{n}\right)\right) \in r$ for all $\left(x_{1}, \ldots, x_{n}\right) \in r^{D(\mathrm{~A})}$. A map

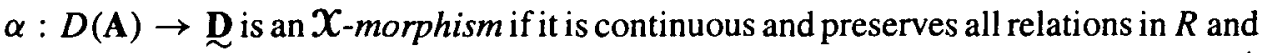
the graphs of all (partial) operations in $G \cup H$. The dual $D(\mathbf{A})$ is a substructure of ${\underset{\mathbf{D}}{ }}^{A}$ and hence as a member of $\mathcal{X}$. Similarly, for all $\mathbf{X} \in \mathcal{X}$, the homset $E(\mathbf{X}):=\mathcal{X}(\mathbf{X}, \underset{\sim}{\mathbf{D}})$ of all $\boldsymbol{X}$-morphisms from $\mathbf{X}$ into $\underset{\sim}{\mathbf{D}}$ is a subalgebra of $\underline{\mathbf{D}}^{X}$ and so is a member of $\mathcal{D}$.

The maps $D$ and $E$ have natural extensions to the morphisms in $\mathcal{D}$ and $\mathcal{X}$. The resulting (hom-)functors $D: \mathcal{D} \rightarrow X$ and $E: X \rightarrow \mathcal{D}$ are contravariant and dually adjoint. Moreover, for any $\mathbf{A} \in \mathcal{D}$ we have a natural homomorphism $e_{A}: \mathbf{A} \rightarrow$ $E D(\mathbf{A})$ given by evaluation,

$$
e_{A}(a)(x)=x(a) \quad \text { for all } a \in A \text { and } x \in D(\mathbf{A}),
$$

which is an embedding. We say that $\mathrm{D}$ (or $G \cup H \cup R$ ) yields a natural duality on an algebra $\mathbf{A} \in \mathcal{D}$ if the embedding $e_{A}$ is an isomorphism, that is, the evaluation maps $e_{A}(a)$, for $a \in A$, are the only morphisms from $D(\mathbf{A})$ to $\underset{\sim}{\mathbf{D}}$. We say that $\underset{\sim}{\mathbf{D}}$ (or $G \cup H \cup R$ ) yields a natural duality on $\mathcal{D}$ (based on $\underline{D}$ ) if it yields a duality on every $\mathbf{A} \in \mathcal{D}$. If End $(\underline{D})$ yields a duality on $\mathcal{D}$, we say that $\underline{\mathbf{D}}$ is endodualisable and if $\operatorname{End}(\underline{D}) \cup\{s\}$ yields a duality on $\mathcal{D}$, we say that $\underline{\mathbf{D}}$ is almost endodualisable (with 
extra relation s). If $\underset{\sim}{\mathbf{D}}=(D ; G, H, R, \mathscr{T})$ yields a duality on $\mathcal{D}$ and if the removal of any element of $G \cup H \cup R$ gives a structure which does not yield a duality on $\mathcal{D}$, then we say that $\underset{\sim}{\mathrm{D}}$ yields an optimal duality on $\mathcal{D}$. If $G \cup H \cup R$ is finite we may remove one relation at a time until an optimal duality is obtained. We refer the reader to Davey [4] for an introductory survey on the theory of natural dualities and to the monograph Clark and Davey [2] for a full account.

Assume that $\underline{\mathbf{M}}$ is a finite algebra in $\mathcal{D}$ that has $\underline{\mathbf{D}}$ as a subalgebra. It follows at once that $\underline{\mathbf{M}}$ also generates the quasi-variety $\mathcal{D}$. Given a structure $\underset{\sim}{\mathbf{D}}$ which yields an optimal duality on $\mathcal{D}$ based on $\underline{\mathbf{D}}$, we wish to convert it to a structure $\mathbf{M}$ which yields an optimal duality on $\mathcal{D}$ based on $\underline{\mathbf{M}}$. The idea of transferring a known duality for a finite algebra $\underline{\mathbf{D}}$ to a new duality for another finite algebra $\underline{\mathbf{M}}$ originated in the piggyback techniques developed in Davey and Werner $[15,16]$. A powerful method for transferring dualities within the same quasi-variety of algebras via retractions, known as the 'Siena method', was presented in Davey [5]. This was later generalised to the Subalgebra Duality Transfer Theorem by Saramago [23]. In order to discuss these results, we require some notation.

Let $\underline{\mathbf{D}}$ be a finite algebra. Let $\underline{\mathbf{M}}$ be a finite algebra in $\mathcal{D}:=\mathbb{I S P}(\underline{D})$ that has $\underline{\mathbf{D}}$ as a subalgebra. Thus, $\mathcal{D}=\mathbb{D S P}(\underline{\mathbf{M}})$. Let $r$ be an $n$-ary algebraic relation on $\underline{\mathbf{D}}$ and let $\mathbf{r}$ be the corresponding subalgebra of $\underline{\mathbf{D}}^{n}$. Note that, since $\mathbf{r} \leqslant \underline{\mathrm{D}}^{n}$ and $\underline{\mathrm{D}}^{n} \leqslant \underline{\mathbf{M}}^{n}$, we may view $r$ as an $n$-ary algebraic relation on $\underline{\mathbf{M}}$. Following the notation used in [5] and [2], this relation on $\underline{\mathbf{M}}$ is denoted by $r_{D}$. Similarly, if $g$ is an algebraic total (or partial) operation on $D$ then $g_{D}$ denotes $g$ regarded as an algebraic partial operation on $M$. If $\underset{\sim}{\mathbf{D}}=\langle D ; G, H, R, \mathscr{T}\rangle$ is algebraic over $\underline{\mathbf{D}}$, then the corresponding sets of algebraic partial operations and relations on $\underline{\mathbf{M}}$ are denoted by

$$
G_{D}:=\left\{g_{D} \mid g \in G\right\}, H_{D}:=\left\{h_{D} \mid h \in H\right\} \text { and } R_{D}:=\left\{r_{D} \mid r \in R\right\} .
$$

The ability to transfer a duality for $\mathcal{D}$ based on $\underline{D}$ to one based on $\underline{M}$ relies on the existence of a set $\Omega=\left\{\omega_{1}, \ldots, \omega_{k}\right\}$ of endomorphisms of $\underline{\mathbf{M}}$ which satisfy:

(E1) $\omega_{i}(M) \subseteq D$ for all $i$, and

(E2) $\omega_{1}, \ldots, \omega_{k}$ separate the points of $M$.

These conditions guarantee that the product map $\omega_{1} \sqcap \cdots \sqcap \omega_{k}: \underline{\mathbf{M}} \rightarrow \underline{\mathbf{D}}^{k}$ is an embedding. Our assumptions that $\underline{\mathbf{D}}$ is a subalgebra of $\underline{\mathbf{M}}$ and that $\underline{\mathbf{M}}$ belongs to $\mathcal{D}$ guarantee the existence of such a set of endomorphisms. Indeed, if $\omega: \underline{\mathbf{M}} \rightarrow \underline{\mathbf{D}}^{k}$ is an embedding and $\pi_{i}: \underline{\mathbf{D}}^{k} \rightarrow \underline{\mathbf{D}}$ is the $i$ th projection, then we can choose $\Omega=$ $\left\{\pi_{1} \circ \omega, \ldots, \pi_{k} \circ \omega\right\}$.

THEOREM 1.1 (Subalgebra Duality Transfer Theorem [23, Proposition 2.1]). Let $\underline{\mathbf{M}}$ be a finite algebra in $\mathcal{D}:=\mathbb{N S P}(\underline{\mathbf{D}})$. Assume that $\underline{\mathbf{D}}$ is a subalgebra of $\underline{\mathrm{M}}$ and that $\Omega$ is a subset of $\operatorname{End}(\underline{\mathbf{M}})$ that satisfies (E1) and (E2). If $\underline{\mathbf{D}}=\langle D ; G, H, R, \mathscr{T}\rangle$ 
yields a duality on $\mathcal{D}$ based on $\underline{\mathbf{D}}$ then $\mathbb{\mathcal { M }}:=\left\langle M ; \Omega, G_{D} \cup H_{D}, R_{D} \cup\{D\}, \mathscr{T}\right\rangle$ yields a duality on $\mathcal{D}$ based on $\underline{\mathrm{M}}$.

REMARKS 1.2. Henceforth, given an algebraic relation $r$ on $\underline{\mathbf{D}}$ we shall drop the subscript from $r_{D}$ when regarding $r$ as a relation on $\underline{\mathbf{M}}$. It will always be clear from the context which hat $r$ is wearing. Theorem 1.1 remains valid if we replace the assumption that $\underline{\mathbf{D}}$ is a subalgebra of $\underline{\mathbf{M}}$ by the assumption that there is an embedding $\nu: \underline{\mathbf{D}} \rightarrow \underline{\mathbf{M}}$. Simply replace $D$ by $v(D)$ throughout the statement of the theorem. Similarly, all of our results that assume that $\underline{\mathbf{M}}$ has $\underline{\mathbf{D}}$ as a subalgebra remain valid under the weaker assumption that $\underline{\mathbf{M}}$ has a subalgebra isomorphic to $\underline{\mathbf{D}}$.

The Subalgebra Duality Transfer Theorem was first presented, under the assumption that $\underline{\mathbf{M}}$ has $\underline{\mathbf{D}}$ as a subretract, in [5]. We recall that $\underline{\mathbf{D}}$ is a retract of $\underline{\mathbf{M}}$ if there are homomorphisms $u: \underline{\mathbf{D}} \rightarrow \underline{\mathbf{M}}$ and $v: \underline{\mathbf{M}} \rightarrow \underline{\mathbf{D}}$ such that $v \circ u=\mathrm{id}_{b}$ in which case $v$ is called a retraction and $u$ is called a coretraction. In the case that $\underline{D}$ is a subalgebra of $\underline{\mathbf{M}}$ and $u$ is simply the inclusion map, we say that $\underline{\mathbf{D}}$ is a subretract of $\underline{\mathbf{M}}$ and refer to $v$ as a subretraction. Retractions will play a vital role in our theory.

The concept of entailment is central to the process of obtaining an optimal duality from a non-optimal one. We shall develop the theory of entailment based on the algebra $\underline{\mathbf{D}}$, but in practice will also need the corresponding ideas and results based on $\underline{\text { M. Let }} S \cup\{s\}$ be a set of finitary algebraic (partial) operations and relations on $\underline{\mathbf{D}}$ and let $\mathbf{A}$ belong to $\mathcal{D}:=\mathbb{D S P}(\underline{\mathbf{D}})$. We say that $S$ entails $s$ on $D(\mathbf{A})=\mathcal{D}(\mathbf{A}, \underline{\mathbf{D}})$ if every map $\alpha: D(\mathbf{A}) \rightarrow D$ that preserves the (partial) operations and relations in $S$ also preserves $s$. We say that $S$ entails $s$ if $S$ entails $s$ on $D(\mathbf{A})$ for all $\mathbf{A} \in \mathcal{D}$. An important fact connecting entailment with duality, which will be used often below, is the following Test Algebra Lemma.

Lemma 1.3 (Test Algebra Lemma, [13, Lemma 2.3, Proposition 2.5]). Let $R \cup\{s\}$ be a family of finitary algebraic (partial) operations and relations on a finite algebra $\underline{\mathbf{D}}$ and let $\mathcal{D}:=\mathbb{I S P}(\underline{\mathrm{D}})$.

(i) $R$ entails $s$ if and only if $R$ entails $s$ on $D(s)=\mathcal{D}(\mathbf{s}, \underline{D})$.

(ii) If $R$ yields a duality (based on $\underline{\mathrm{D}}$ ) on the algebra $\mathrm{s}$, then $R$ entails $s$.

(iii) Assume that $R$ yields a duality on $\mathcal{D}$ (based on $\underline{\mathrm{D}}$ ) and let $r \in R$. Then the following are equivalent:

(a) $R \backslash\{r\}$ yields a duality on $\mathcal{D}$;

(b) $R \backslash\{r\}$ yields a duality on the algebra $\mathbf{r}$;

(c) $R \backslash\{r\}$ entails $r$ on $D(\mathbf{r})=\mathcal{D}(\mathbf{r}, \underline{\mathbf{D}})$.

Hence, if $G \cup H \cup R$ yields a duality on $\mathcal{D}$, we may delete a relation or (partial) operation $s$ from $G \cup H \cup R$ without destroying the duality if and only if $(G \cup H \cup R) \backslash\{s\}$ 
entails $s$. If $h$ is an $n$-ary algebraic (partial) operation on $\underline{\mathbf{D}}$ then graph $(h)$ is an $(n+1)$ ary algebraic relation on $\underline{D}$ and the corresponding subalgebra of $\underline{D}^{n+1}$ will be denoted by $\mathbf{h}$. (Since $S$ entails $s$ on $D(\mathbf{A})$ if and only if $S$ entails $s$ on $D(\mathbf{B})$ for each algebra $\mathbf{B} \in \mathcal{D}$ with $\mathbf{B} \cong \mathbf{A}$, we could just as well define $\mathbf{h}$ to be the subalgebra of $\underline{\mathbf{D}}^{n}$ corresponding to $\operatorname{dom}(h)$.)

Our aim in this paper is to provide a method for transferring optimal dualities. Because of the important role played by the endomorphisms of $\underline{\mathbf{M}}$ in the Subalgebra Duality Transfer Theorem and because we are usually happy to include (a generating set for) the endomorphism monoid on $\underline{\mathbf{M}}$ in the dualising structure for $\underline{\mathbf{M}}$, we shall concentrate here on dualities which are optimal modulo endomorphisms. That is, we shall seek a set $S$ of finitary algebraic relations on $\underline{\mathbf{M}}$ such that $\operatorname{End}(\underline{\mathbf{M}}) \cup S$ yields a duality on $\mathcal{D}$ based on $\underline{\mathbf{M}}$ but if any member of $S$ is removed the resulting structure does not yield a duality on $\mathcal{D}$.

After transferring an optimal duality from a (usually small) algebra $\underline{\mathbf{D}}$ up to a bigger algebra $\underline{\mathbf{M}} \in \mathcal{D}$ by the Duality Transfer Theorem 1.1, it has been far from clear which reducts of a given dualising structure $\underset{\sim}{\mathbf{M}}$ yield optimal (modulo endomorphisms) dualities on $\mathcal{D}$ based on $\underline{\mathbf{M}}$. Only one thing was known for sure: if End $(\underline{M}) \cup S$ yields a duality on $\mathcal{D}$ based on $\underline{\boldsymbol{M}}$, then a relation $s \in S$ can be removed without destroying the duality provided that the corresponding algebra $\mathbf{s}$ is a retract of $\underline{\mathbf{M}}$. This follows from the following fact from [12, Lemmas 4.1, 4.2], which we state with respect to $\underline{D}$ but use with respect to both $\underline{\mathbf{D}}$ and $\underline{\mathbf{M}}$ : the set $\operatorname{End}(\underline{\mathbf{D}})$ yields a duality (based on $\underline{\mathbf{D}}$ ) on every retract of the algebra $\underline{\mathbf{D}}$.

The set of all finitary algebraic relations on a fixed finite algebra is usually denoted by $\mathscr{B}$ and is referred to as the brute force set of relations as it is the largest set of relations that could be used to obtain a duality and, moreover, if any set of relations yields a duality then $\mathscr{B}$ does. Since we need to distinguish the particular algebra on which we are basing our duality, typically $\underline{\mathbf{D}}$ or $\underline{\mathbf{M}}$, we define

$$
\mathscr{B}(\underline{\mathbf{D}}):=\{r \mid r \text { is an } n \text {-ary algebraic relation on } \underline{\mathbf{D}} \text { for some } n \in \mathbb{N}\} \text {. }
$$

We reiterate that if $\underline{\mathbf{D}}$ is a subalgebra of $\underline{\mathbf{M}}$ then $\mathscr{B}(\underline{D}) \subseteq \mathscr{B}(\underline{\mathbf{M}})$ since each subalgebra of a power of $\underline{\mathbf{D}}$ is a subalgebra of a power of $\underline{\mathbf{M}}$ (see Remarks 1.2).

According to the Test Algebra Lemma, $R$ does not entail $s$ if and only if there exists a map $\gamma^{\prime}: \mathcal{D}(\mathbf{s}, \underline{\mathbf{D}}) \rightarrow D$ such that the set

$$
\text { Fail } \frac{\mathbf{D}}{s}\left(\gamma^{\prime}\right):=\left\{r \in \mathscr{B}(\underline{\mathbf{D}}) \mid \gamma^{\prime}: \mathcal{D}(\mathbf{s}, \underline{\mathbf{D}}) \rightarrow D \text { fails to preserve } r\right\},
$$

contains $s$ but contains no member of $R$. We often work within some fixed subset $\mathscr{R}$ of $\mathscr{B}(\underline{D})$. Then the set Fail $\frac{\mathbf{D}}{s}\left(\gamma^{\prime}\right) \cap \mathscr{R}$ is called a failset of $s$ (within $\mathscr{R}$ ) provided it contains $s$. We refer to $U \subseteq \mathscr{R}$ as a failset (within $\mathscr{R}$ ) if $U$ is a failset of some $s \in \mathscr{R}$. Let $\mathscr{F}_{s}$ be the set of all failsets of $s$ (within $\mathscr{R}$ ) and let $\mathscr{F}:=\bigcup\left\{\mathscr{F}_{s} \mid s \in \mathscr{R}\right\}$. Order 
both $\mathscr{F}_{s}$ and $\mathscr{F}$ by set inclusion. Minimal elements of $\mathscr{F}_{s}$ are called locally minimal failsets (within $\mathscr{R}$ ) while minimal elements of $\mathscr{F}$ are called globally minimal failsets (within $\mathscr{R}$ ). When working simultaneously with algebras $\underline{\mathbf{D}}$ and $\underline{\mathbf{M}}$ we add a prefix and refer to $\underline{\mathbf{D}}$-failsets and $\underline{\mathbf{M}}$-failsets. The following result, which will be required in the final section of the paper, is extracted from a characterisation of globally minimal failsets given in Davey and Priestley [13, Theorem 4.2].

THEOREM 1.4. Let $\underline{\mathbf{D}}$ be a finite algebra and let $\mathcal{D}:=\mathbb{N S P}(\underline{\mathrm{D}})$. Assume that $\mathscr{R} \subseteq \mathscr{B}(\underline{D})$ yields a duality on $\mathcal{D}$ (based on $\underline{\mathbf{D}}$ ) and let $U \subseteq \mathscr{R}$. Then the following are equivalent:

(a) $U$ is a globally minimal failset within $\mathscr{R}$;

(b) $U$ is a locally minimal failset of $r$ within $\mathscr{R}$, for all $r \in U$;

(c) $\mathscr{R} \backslash U$ does not yield a duality on $\mathcal{D}$ but $(\mathscr{R} \backslash U) \cup\{r\}$ yields a duality on $\mathcal{D}$ for all $r \in U$;

(d) for all $r \in U$,

(i) $\mathscr{R} \backslash U$ does not yield a duality on $\mathbf{r}$, and

(ii) $(\mathscr{R} \backslash U) \cup\{r\}$ yields a duality on $U$.

For discussion of the theory of entailment, failsets and optimal dualities beyond the bare details given here we refer to $[8,11,13,22,24]$ and to [2, Chapters 2,8 and 9].

The Test Algebra Lemma 1.3 says that a relation $r$ can be removed from a dualising set $R$ of relations without destroying the duality provided $R \backslash\{r\}$ yields a duality on the single algebra $\mathbf{r}$, which therefore has been called a test algebra. Modifying the concept slightly, throughout this paper an algebra s, corresponding to an algebraic relation $s$ will be said to be a test algebra (for showing that $R$ does not yield a duality on $\mathcal{D}$ ) if $R$ does not yield a duality on the algebra $\mathbf{s}$. The Retraction Test Algebra Lemma from Haviar and Priestley ([20, Lemma 4]; see also [4, Lemma 6.3] and [13, Lemma 4.2]) tells us that a known test algebra $\mathbf{s}$ can be replaced by any algebra $\mathbf{t}$ that retracts onto $\mathbf{s}$. Its corollary ([20, Corollary 5]) says that if $\mathbf{s}$ is a test algebra showing non-endodualisability of $\underline{\mathbf{M}}$ and if $\mathbf{s}$ is a retract of the $k$-generated free algebra $\mathbf{F D}(k)$ (or equivalently, if $\mathbf{s}$ is a $k$-generated projective algebra in $\mathcal{D}$ ), then $\underline{\mathbf{M}}$ is not $k$-endoprimal.

In Section 2 we present our main result, the Optimal Duality Transfer Theorem, which can be described as follows. Assume that an optimal duality for $\mathcal{D}$ based on $\underline{\mathbf{D}}$ is given by some 'minimal' relations and that this structure has been transferred to a duality for $\mathcal{D}$ based on $\underline{\mathbf{M}}$. Using the Test Algebra Lemma we may remove from the transferred duality all the relations $r$ such that $\mathbf{r}$ is a retract of $\underline{\mathbf{M}}$. Our theorem states that the resulting duality for $\mathcal{D}$ based on $\underline{\mathbf{M}}$ is already optimal modulo endomorphisms. Hence a 'minimal' test algebra with respect to $\underline{\mathbf{D}}$ can be transferred to a test algebra with respect to $\underline{\mathbf{M}}$ provided it is not a retract of $\underline{\mathbf{M}}$. 
In Section 3 we assume that the algebra $\underline{\mathbf{D}}$ we start with is almost endodualisable but not endodualisable. Hence the duality transferred onto any $\underline{\mathbf{M}}$ having $\underline{\mathbf{D}}$ as a subretract is given by $\operatorname{End}(\mathbf{M})$ plus one extra relation. We show that this extra relation determines the unique globally minimal $\underline{\mathbf{M}}$-failset disjoint from $\operatorname{End}(\underline{\mathbf{M}})$ within $\mathscr{R}:=\operatorname{End}(\underline{\mathbf{M}}) \cup \mathscr{B}(\underline{\mathbf{D}})$.

In Section 4 we present some applications of the results of Section 2 and Section 3. We first apply the results of Section 2 to transfer optimal dualities from the three-element Kleene algebra to derive new optimal dualities for finite Kleene algebras having fixpoints. Then we illustrate the results of Section 3 in the varieties of semilattices, bounded distributive lattices and Stone algebras.

The final section of the paper contains the proofs of the technical lemmas required in Section 2 to establish our Optimal Duality Transfer Theorem.

\section{Transferring optimal dualities: theory}

Let $\underline{\mathbf{D}}$ be a finite algebra and assume that the topological structure

$$
\underline{\mathbf{D}}=\langle D ; \operatorname{End}(\underline{\mathbf{D}}), S, \mathscr{T}\rangle
$$

yields a duality on $\mathcal{D}=\mathbb{U S P}(\underline{D})$ which is optimal modulo endomorphisms. In practice, we would like our dualising structure $\mathbf{D}$ to be optimally optimal. For example, we might want the relations $s \in S$ to have minimum cardinality (or minimum arity or both). In order to understand these ideas better, we shall now discuss optimal sets of relations and their interaction with failsets in more detail.

Let $R$ be a set of finitary algebraic relations on $\underline{\mathbf{D}}$ and let $s \in R$. A relation $s_{1} \in \mathscr{B}(\underline{D})$ is a substitute for $s$ relative to $R$ if $(R \backslash\{s\}) \cup\left\{s_{1}\right\}$ entails $s$. Thus, if $R$ yields a duality on $\mathcal{D}$ and $s_{\mathrm{I}}$ is a substitute for $s \in R$, then $(R \backslash\{s\}) \cup\left\{s_{1}\right\}$ also yields a duality on $\mathcal{D}$. Nevertheless, the duality given by $(R \backslash\{s\}) \cup\left\{s_{1}\right\}$ may not be optimal even when the duality given by $R$ is. We shall say that $s$ is needed in $R$ if $R \backslash\{s\}$ does not entail $s$ and that a subset $S$ of $R$ is optimal in $R$ if every relation $s \in S$ is needed in $R$. If every relation $s \in R$ is needed in $R$, we say that $R$ is optimal. Thus $R$ yields an optimal duality on $\mathcal{D}$ if and only if $R$ yields a duality on $\mathcal{D}$ and $R$ is optimal. Note that if $s$ is needed in $R$ and $s_{1}$ is a substitute for $s$ relative to $R$, then $s_{1}$ is needed in $(R \backslash\{s\}) \cup\left\{s_{1}\right\}$. By the Test Algebra Lemma 1.3, the relation $s$ is needed in $R$ if and only if $R \backslash\{s\}$ does not entail $s$ on $D(\mathbf{s})$, that is, there exists a map $\gamma^{\prime}: \mathcal{D}(\mathbf{s}, \underline{\mathbf{D}}) \rightarrow D$ such that

(N1) $\gamma^{\prime}$ does not preserve $s$, that is, $s \in$ Fail $\frac{\mathbf{p}}{\mathbf{s}}\left(\gamma^{\prime}\right)$,

(N2) $\gamma^{\prime}$ preserves each relation $r \in R \backslash\{s\}$, that is, Fail $\frac{\mathbf{D}}{\mathbf{s}}\left(\gamma^{\prime}\right) \cap(R \backslash\{s\})=\varnothing$.

Let $\underline{\mathbf{M}}$ be a finite algebra in $\mathcal{D}$. We shall say that the relation $s$ avoids $\underline{\mathbf{M}}$ relative to $R$ if there exists a map $\gamma^{\prime}: \mathcal{D}(\mathbf{s}, \underline{\mathrm{D}}) \rightarrow D$ which satisfies (N1), (N2) and 
(A) $\gamma^{\prime}$ preserves every finitary algebraic relation $r \in \mathscr{B}(\mathbf{D})$ such that $\mathbf{r} \cong \underline{\mathbf{M}}$, that is, Fail $\frac{\mathbf{D}}{\mathbf{s}}\left(\gamma^{\prime}\right) \cap\{r \in \mathscr{B}(\underline{\mathbf{D}}) \mid \mathbf{r} \cong \underline{\mathbf{M}}\}=\varnothing$.

If $S \subseteq R$ and each $s \in S$ avoids $\underline{\mathbf{M}}$ relative to $R$ then we say that $S$ avoids $\underline{\mathbf{M}}$ relative to $R$. In the case that $S=R$, we say simply that $R$ avoids $\underline{\mathbf{M}}$. We often prove that $s$ avoids $\underline{\mathbf{M}}$ relative to $R$ by establishing a stronger minimality condition on $s$. We say that $s$ is as small as possible relative to $R$ if there exists a map $\gamma^{\prime}: \mathcal{D}(\mathbf{s}, \underline{\mathrm{D}}) \rightarrow D$ which satisfies (N1), (N2) and

(S) $\gamma^{\prime}$ preserves every relation $s_{1} \in \mathscr{B}(\underline{D})$ such that $s$ is not a retract of $s_{1}$, that is, Fail $\frac{\mathbf{D}}{\mathbf{s}}\left(\gamma^{\prime}\right) \subseteq\left\{s_{1} \in \mathscr{B}(\underline{\mathbf{D}}) \mid \mathbf{s}\right.$ is a retract of $\left.\mathbf{s}_{1}\right\}$.

The following lemma collects together some simple but useful connections between the concepts introduced above.

LEMMA 2.1. Let $\underline{\mathbf{D}}$ be a finite algebra and let $\underline{\mathbf{M}}$ be a finite algebra in $\mathcal{D}:=\mathbb{S P}(\mathbf{D})$. Assume that $R$ is a set of finitary algebraic relations on $\underline{\mathbf{D}}$ and let $s \in R$.

(i) If $s$ is as small as possible relative to $R$ and $s_{1}$ is a substitute for $s$ relative to $R$, then $\mathbf{s}$ is a retract of $\mathbf{s}_{1}$ and therefore, in particular, $|s| \leqslant\left|s_{1}\right|$.

(ii) If $s$ is as small as possible relative to $R$ and $\mathbf{s}$ is not a retract of $\underline{\mathbf{M}}$, then $s$ avoids $\mathbf{M}$ relative to $R$.

(iii) If $s$ avoids $\underline{\mathbf{M}}$ relative to $R$, then no substitute for $s$ relative to $R$ can be isomorphic qua algebra to $\underline{\mathbf{M}}$.

(iv) If $s$ is as small as possible relative to $R$ or if $s$ avoids $\underline{\mathbf{M}}$ relative to $R$, then $s$ is needed in $R$.

By (i), one way to guarantee that the set $R$ is optimally optimal is to ensure that every relation $s$ in $R$ is as small as possible relative to $R$.

The following two results, which are proved in Section 5 , provide sufficient conditions under which a $\underline{\mathbf{D}}$-failset of an algebraic relation $s$ on $\underline{\mathbf{D}}$ may be transferred to a very closely related $\underline{\mathbf{M}}$-failset of the relation $s$ on $\underline{\mathbf{M}}$.

LEMMA 2.2. Let $\underline{\mathbf{D}}$ be a finite algebra, let $\mathcal{D}:=\mathbb{B} \mathbb{P}(\underline{\mathrm{D}})$ and assume that $\underline{\mathbf{M}} \in \mathcal{D}$ is finite and has $\underline{\mathbf{D}}$ as a subalgebra. Let $s$ and $t$ be finitary algebraic relations on $\underline{\mathbf{D}}$ and assume that neither $\underline{\mathbf{D}}$ nor $\underline{\mathbf{M}}$ has $\mathbf{t}$ as a retract. If $\{r \in \mathscr{B}(\underline{D}) \mid \mathbf{t}$ is a retract of $\mathbf{r}\}$ contains a $\underline{\mathbf{D}}$-failset $U$ of $s$, then there exists an $\underline{\mathbf{M}}$-failset $V$ of $s$ such that

(i) $V$ contains no (graphs of) endomorphisms of $\underline{\mathbf{M}}$ and

(ii) $V \cap \mathscr{B}(\underline{D})=U$.

LEMMA 2.3. Let $\underline{\mathbf{D}}$ be a finite algebra, let $\mathcal{D}:=\mathbb{N S P}(\underline{\mathbf{D}})$ and assume that $\underline{\mathbf{M}} \in \mathcal{D}$ is finite and has $\underline{\mathbf{D}}$ as a subalgebra. Let $S$ be a set of finitary algebraic relations on $\underline{\mathbf{D}}$ and let $s \in S$. The following conditions are related by $(1) \Rightarrow(2) \Rightarrow(3)$ :

(1) $s$ (as a relation on $\underline{\mathbf{D}}$ ) avoids $\underline{\mathbf{M}}$ relative to $\operatorname{End}(\underline{\mathbf{D}}) \cup S$; 
(2) $s$ (as a relation on $\underline{\mathbf{M}}$ ) is needed in $\mathrm{End}(\underline{\mathrm{M}}) \cup S \cup\{D\}$;

(3) $\mathbf{s}$ is not a retract of $\underline{\mathbf{M}}$.

With this technical lemma in hand we are ready to prove the main result of this section.

THEOREM 2.4 (Optimal Duality Transfer Theorem). Let $\underline{\mathbf{D}}$ be a finite algebra, let $\mathcal{D}:=\mathbb{U S P}(\underline{D})$ and assume that $\underline{\mathbf{M}} \in \mathcal{D}$ is finite and has $\underline{\mathbf{D}}$ as a subalgebra. Let $S$ be a set of finitary algebraic relations on $\underline{\mathbf{D}}$ and assume that the structure $\underline{\mathbf{D}}=\langle D ; \operatorname{End}(\underline{\mathbf{D}}), S, \mathscr{T}\rangle$ yields a duality on $\mathcal{D}$ based on $\underline{\mathbf{D}}$ which is optimal modulo endomorphisms. Define $S^{\circ}:=\{s \in S \mid \mathbf{s}$ is not a retract of $\underline{\mathbf{M}}\}$. If $S^{\circ}$ avoids $\underline{\mathbf{M}}$ relative to $\operatorname{End}(\mathrm{D}) \cup S^{\circ}$, then, with the possible exception that $D$ may not be needed, $\underset{\mathbf{M}}{\sim}:=\left\langle M ; \operatorname{End}(\underline{\mathbf{M}}), S^{\circ} \cup\{D\}, \mathscr{T}\right\rangle$ yields a duality on $\mathcal{D}$ based on $\underline{\mathbf{M}}$ which is optimal modulo endomorphisms. Moreover, if $\underline{\mathrm{D}}$ is a subretract of $\underline{\mathrm{M}}$, then $D$ is not needed and consequently $\underset{\sim}{\mathbf{M}}:=\left\langle M ; \operatorname{End}(\underline{\mathbf{M}}), S^{\circ}, \mathscr{T}\right\rangle$ yields a duality on $\mathcal{D}$ based on $\underline{\mathbf{M}}$ which is optimal modulo endomorphisms.

Proof. From Theorem 1.1, it follows that $\mathbf{M}^{\prime}=\langle M ; \operatorname{End}(\underline{\mathbf{M}}), S \cup\{D\}, \mathscr{T}\rangle$ yields

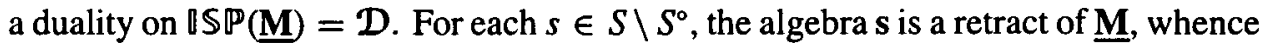
End $(\underline{\mathbf{M}})$ entails $s$. Hence the relations $s \in S \backslash S^{\circ}$ can be removed from $\underline{\mathbf{M}}^{\prime}$ without destroying the duality. Thus, $\underset{\sim}{\mathbf{M}}=\left\langle M\right.$; End $\left.(\underline{\mathbf{M}}), S^{\circ} \cup\{D\}, \mathscr{T}\right\rangle$ yields a duality on $\square \mathbb{S P}(\underline{\mathbf{M}})$. If $u: \underline{\mathbf{M}} \rightarrow \underline{\mathbf{D}}$ is a subretraction, then $u \in \operatorname{End}(\underline{\mathbf{M}})$ with $\operatorname{fix}(u)=D$, whence $u$ entails $D$. The result now follows from the previous lemma.

We now give some natural conditions on failsets that will ensure that $s$ avoids $\underline{M}$ relative to End(D) $\cup S$. We almost always use these lemmas when applying the Optimal Duality Transfer Theorem. Recall that a subset $U$ of $\mathscr{B}(\mathbf{D})$ is a $\underline{\mathbf{D}}$-failset of $s$ if $s \in U$ and there exists a map $\gamma^{\prime}: \mathcal{D}(\mathbf{s}, \underline{\mathbf{D}}) \rightarrow D$ such that Fail $\frac{\mathbf{D}}{\mathbf{s}}\left(\gamma^{\prime}\right)=U$.

LEMMA 2.5. Let $\underline{\mathbf{D}}$ be a finite algebra, let $\mathcal{D}:=\mathbb{B S P}(\underline{\mathbf{D}})$ and assume that $\underline{\mathbf{M}}$ is a finite algebra in $\mathcal{D}$. Let $S$ be a set of finitary algebraic relations on $\underline{\mathrm{D}}$, let $s \in S$ and consider the following conditions:

(1) $\left\{s_{1} \in \mathscr{B}(\underline{D}) \mid \mathrm{s}\right.$ is a retract of $\left.\mathbf{s}_{1}\right\}$ contains a $\mathbf{D}$-failset of $s$;

(2) $s$ is as small as possible relative to $S$;

(3) $s$ is as small as possible relative to $\operatorname{End}(\mathbf{D}) \cup S$;

(4) $s$ avoids $\underline{\mathbf{M}}$ relative to $\operatorname{End}(\underline{D}) \cup S$.

If $\mathbf{s}$ is not a retract of $\underline{\mathbf{M}}$ then (3) $\Rightarrow$ (4). If $\mathbf{s}$ is not a retract of $\underline{\mathbf{D}}$ then (2) $\Rightarrow(3)$. If $\mathbf{s}$ is not a retract of $\mathbf{r}$ for all $r \in S \backslash\{s\}$, then $(1) \Rightarrow$ (2).

Proof. If $\mathbf{s}$ is not a retract of $\underline{\mathbf{M}}$, then (3) implies (4) by Lemma 2.1 (ii). Assume that $\mathbf{s}$ is not a retract of $\underline{\mathbf{D}}$. If (2) holds, then there exists $\gamma^{\prime}: \mathcal{D}(\mathbf{s}, \underline{\mathbf{D}}) \rightarrow D$ 
satisfying (N1), (N2) and (S) with respect to $R:=S$. Since $s$ is not a retract of $\underline{\mathbf{D}}$, by (S) the map $\gamma^{\prime}$ preserves every relation isomorphic qua algebra to $\underline{\mathbf{D}}$. Thus, $\gamma^{\prime}$ preserves graph(e) and consequently preserves $e$, for all $e \in \operatorname{End}(\mathbf{D})$. It follows at once that $\gamma^{\prime}$ satisfies (N1), (N2) and (S) with respect to $R:=\operatorname{End}(\mathrm{D}) \cup S$, whence $s$ is as small as possible relative to End(D) $\cup S$. Hence, (2) implies (3). Finally, assume that $\mathbf{s}$ is not a retract of $\mathbf{r}$ for all $r \in S \backslash\{s\}$. It then follows from (1) that there is a map $\gamma^{\prime}: \mathcal{D}(\mathbf{s}, \underline{\mathbf{D}}) \rightarrow D$ such that (N1), (N2) and (S) hold with respect to $R:=S$, whence $s$ is as small as possible relative to $S$. Hence, (1) implies (2).

The lemma above is typically applied to algebraic relations $s$ that have a subalgebra isomorphic to $\underline{\mathbf{D}}$. Our next lemma applies to 'small' algebraic relations that do not have a subalgebra isomorphic to $\underline{\mathbf{D}}$.

LEMMA 2.6. Let $\underline{\mathbf{D}}$ be a finite algebra, let $\mathcal{D}:=\mathbb{U} S \mathbb{P}(\underline{\mathrm{D}})$ and assume that $\underline{\mathrm{M}} \in \mathcal{D}$ is finite and has $\underline{\mathbf{D}}$ as a subalgebra. Let $S$ be a set of finitary algebraic relations on $\underline{\mathrm{D}}$ and let $s \in S$. Assume that $\mathrm{s}$ has no subalgebra isomorphic to $\underline{\mathrm{D}}$ and $\mathrm{r}$ has a subalgebra isomorphic to $\underline{\mathbf{D}}$ for all $r \in S \backslash\{s\}$. If

$$
\{r \in \mathscr{B}(\underline{D}) \mid \mathbf{r} \text { has no subalgebra isomorphic to } \underline{\mathbf{D}}\}
$$

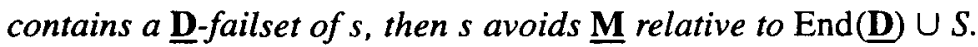

PROOF. Let $U$ be a $\underline{\mathbf{D}}$-failset of $s$ and assume that $\mathbf{r}$ has no subalgebra isomorphic to $\underline{\mathbf{D}}$ for all $r \in U$. Hence, $U \cap S=\{s\}$ and so (N1) and (N2) hold with respect to $R:=S$. If $r \in \mathscr{B}(\underline{D})$ with $\mathbf{r} \cong \underline{\mathbf{M}}$, then $\mathbf{r}$ has a subalgebra isomorphic to $\underline{\mathbf{D}}$ and hence $r \notin U$. Thus, $s$ avoids $\underline{\mathrm{M}}$ relative to $S$. Since $D \notin U$ and since, for all $e \in \operatorname{End}(\mathbf{D})$, the relation graph $(e)$ is isomorphic $q u a$ algebra to $\underline{\mathbf{D}}$, the failset $U$ contains no (graphs of) endomorphisms of $\underline{\mathbf{D}}$. Consequently, the $\underline{\mathbf{D}}$-failset $U$ also shows that $s$ avoids $\underline{\mathbf{M}}$ relative to $\operatorname{End}(\mathbf{D}) \cup S$.

\section{Almost endodualisability}

There are many finite algebras $\underline{\mathbf{D}}$ that are dualised by some endomorphisms of

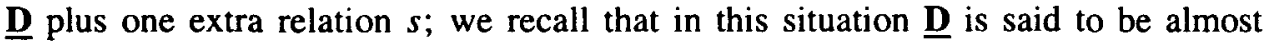
endodualisable. Moreover, in many examples (as we shall see in Section 4 below) the extra relation $s$ satisfies Condition (1) of Lemma 2.5. In this case we obtain the following transfer theorem.

THEOREM 3.1 (Almost Endodualisability Transfer Theorem). Let $\underline{\mathbf{D}}$ be a finite algebra, let $\mathcal{D}:=\mathbb{N} \mathbb{P}(\underline{D})$ and assume that $\underline{\mathrm{M}} \in \mathcal{D}$ is finite and has $\underline{\mathrm{D}}$ as a subretract. Assume that $\underline{\mathrm{D}}$ is almost endodualisable with extra relation $s$. 
(0) $\underline{M}$ is almost endodualisable with extra relation $s$.

If, moreover, $\underline{\mathbf{D}}$ is not endodualisable and $\left\{s_{1} \in \mathscr{B}(\underline{\mathbf{D}}) \mid \mathrm{s}\right.$ is a retract of $\left.\mathbf{s}_{1}\right\}$ contains a

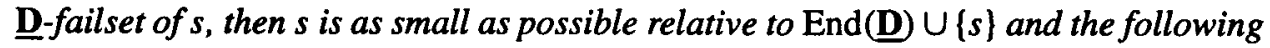
hold

(1) $\underline{M}$ is endodualisable if and only if $s$ is a retract of $\underline{\mathbf{M}}$

(2) if $\underline{\mathrm{M}}$ is non-endodualisable then $\mathbf{s}$, and any finite $\mathbf{t} \in \mathcal{D}$ that has $\mathbf{s}$ as a retract, serves as a test algebra showing that $\underline{\mathrm{M}}$ is not endodualisable,

(3) if $\mathbf{s}$ is a retract of the $k$-generated free algebra $\mathbf{F D}(k)$, then $\underline{\mathbf{M}}$ is $k$-endoprimal if and only if $\mathbf{s}$ is a retract of $\underline{\mathbf{M}}$.

Proof. It follows from Theorem 1.1 that $\underset{\sim}{\mathbf{M}}=\langle M ; \operatorname{End}(\underline{\mathbf{M}}), s, \mathscr{T}\rangle$ yields a duality on $\mathcal{D}$ based on $\underline{\mathbf{M}}$. If $\mathbf{s}$ is a retract of $\underline{\mathbf{M}}$, then $s$ can be removed from $\underline{\mathbf{M}}$ without destroying the duality and so $\underline{M}$ is endodualisable. The non-endodualisability of $\underline{\mathbf{D}}$ implies that $s$ is needed in $\operatorname{End}(\underline{\mathbf{D}}) \cup\{s\}$, so that $\mathbf{s}$ is not a retract of $\underline{\mathbf{D}}$. By Lemma 2.5, $s$ is as small as possible relative to $\operatorname{End}(\mathbf{D}) \cup\{s\}$, and, if $s$ is not a retract of $\underline{\mathbf{M}}$, then $s$ avoids $\underline{\mathbf{M}}$ relative to End $(\underline{D}) \cup\{s\}$. The rest of the proof follows from Theorem 2.4, and from the Retraction Test Algebra Lemma and its corollary ([20, Lemma 4, Corollary 5]), which we discussed near the end of Section 1.

For the remainder of the this section we shall assume that $\underline{\mathbf{D}}$ is a finite almost endodualisable but not endodualisable algebra with extra relation $s$ (whence $s$ is not a retract of $\underline{\mathbf{D}}$ ). Moreover, we shall assume that $s$ is binary. We let $\underline{\mathbf{M}}$ be a finite algebra in $\mathcal{D}:=\mathbb{N S} \mathbb{P}(\underline{\mathbf{D}})$ and assume that $\underline{\mathbf{M}}$ has $\underline{\mathbf{D}}$ as a subretract and does not have $s$ as a retract. We say that $s$ is a retractive projection of an $m$-ary relation $s_{1} \in \mathscr{B}(\mathbf{D})(m \geqslant 2)$ if there exist $i \neq j$ with $1 \leqslant i, j \leqslant m$ such that the projection $p_{i j}: M^{m} \rightarrow M^{2}$ satisfies $p_{i j}\left(s_{1}\right)=s$ and, moreover, there exists a homomorphism $q: \mathrm{s} \rightarrow \mathrm{s}_{1}$ such that $p_{i j} \Gamma_{\mathrm{s}_{1}} \circ q=\mathrm{id}_{\mathrm{s}}$ (see [2, page 275]). It is easy to see that if $s$ is a retractive projection of $s_{1}$, then $s_{1}$ entails $s$. Consider the set

$$
U^{s}:=\{r \in \mathscr{B}(\underline{D}) \mid r \text { has } s \text { as a retractive projection }\} .
$$

This set occasionally turns out to be a failset of $s$.

LEMMA 3.2. Let $s \in \mathscr{B}(\underline{D})$. The following are equivalent:

(a) $U^{s}$ is a locally minimal failset of $s$ within $\mathscr{B}(\mathbf{D})$;

(b) $U^{s}$ is a failset of $s$ within $\mathscr{B}(\mathbf{D})$;

(c) $U^{s}$ contains a failset of $s$ within $\mathscr{B}(\mathbf{D})$.

PRoof. Clearly, (a) $\Rightarrow$ (b) $\Rightarrow$ (c), so assume (c). Let $V$ be any failset of $s$ with $V \subseteq U^{s}$. To prove that $U^{s}$ is a locally minimal failset of $s$ it suffices to show that $U^{s} \subseteq V$. Let $r \in U^{s}$. Since $r$ has $s$ as a retractive projection, $r$ entails $s$. As $s$ belongs to the failset $V$, it follows that $r \in V$ and consequently $U^{s} \subseteq V$, as required. 
Obviously, $U^{s}$ is a subset of $\left\{s_{1} \in \mathscr{B}(\mathbf{D}) \mid \mathbf{s}_{1}\right.$ has $\mathbf{s}$ as a retract $\}$. So if $U^{s}$ is a failset, the Almost Endodualisability Transfer Theorem can be applied; but in this case even more can be said. If $V \subseteq \mathscr{B}(\underline{\mathbf{M}})$, we write $V \cap \operatorname{End}(\underline{\mathbf{M}})=\varnothing$ and say that $V$ is disjoint from End $(\underline{\mathbf{M}})$ provided $V$ contains no graph of an endomorphism of $\underline{\mathbf{M}}$.

THEOREM 3.3. Let $\underline{\mathbf{D}}$ be a finite almost endodualisable but not endodualisable algebra with $s \subseteq D^{2}$ as the extra relation and assume that $U^{s}$ is a $\underline{\mathbf{D}}$-failset of $s$ within $\mathscr{B}(\underline{\mathbf{D}})$. Let $\underline{\mathbf{M}}$ be a finite algebra in $\mathcal{D}:=\mathbb{N} \mathbb{P}(\underline{D})$ that has $\underline{\mathbf{D}}$ as a subretract but does not have $\mathrm{S}$ as a retract.

(1) The algebra $\underline{\mathbf{M}}$ is almost endodualisable: let $r \in \mathscr{B}(\mathbf{D})$, then

$$
\mathbf{M}=\langle M ; \operatorname{End}(\underline{\mathbf{M}}), r, \mathscr{T})
$$

yields a duality on $\mathcal{D}$ based on $\underline{\mathrm{M}}$ if and only if $r \in U^{s}$.

(2) For every $r \in U^{s}$, the algebra $\mathbf{r}$ serves as a test algebra showing that $\underline{\mathbf{M}}$ is not endodualisable.

(3) Let $\mathscr{R}:=\operatorname{End}(\underline{\mathbf{M}}) \cup \mathscr{B}(\underline{D})$. Then

(i) $U^{s}$ is a globally minimal $\underline{\mathbf{M}}$-failset within $\mathscr{R}$,

(ii) $U^{s} \cap \operatorname{End}(\underline{\mathbf{M}})=\varnothing$,

(iii) $U^{s}$ is the only globally minimal $\underline{\mathbf{M}}$-failset within $\mathscr{R}$ which is disjoint from End(M).

Proof. Assume that $\underline{\mathbf{D}}$ is almost endodualisable with $s$ as the extra relation; then, by Theorem 1.1, $\underline{M}$ is also almost endodualisable with $s$ as the extra relation. Let $r \in U^{s}$. Then $r$ has $s$ as a retractive projection and hence $r$ entails $s$, whence the structure $\mathbf{M}=\langle M ; \operatorname{End}(\underline{\mathbf{M}}), r, \mathscr{T}\rangle$ yields a duality on $\mathcal{D}$ based on $\underline{\mathbf{M}}$. This proves sufficiency in (1). Since $U^{s}$ is a $\underline{\mathbf{D}}$-failset of $s$ and since $\mathbf{s}$ is not a retract of $\underline{\mathbf{M}}$, it follows from Theorem 3.1 that for every $r \in U^{s}$, the algebra $r$ is a test algebra showing that $\underline{\mathbf{M}}$ is not endodualisable, whence (2) holds.

Lemma 2.2 with $t=s$ implies that there is an $\underline{\mathbf{M}}$-failset $V$ of $s$ which contains no graphs of endomorphisms of $\underline{\mathbf{M}}$ and satisfies $V \cap \mathscr{B}(\underline{\mathbf{D}})=U^{s}$. Hence, $U^{s}$ is an

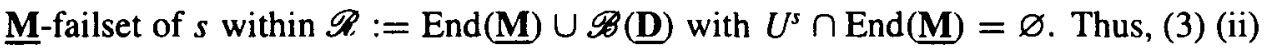
holds. Since any subset of $\mathscr{R}$ that yields a duality on $\mathcal{D}$ (based on $\underline{\mathbf{M}}$ ) must intersect every failset within $\mathscr{R}$ (see [2, Theorem 8.3.1]), the existence of the $\underline{\mathrm{M}}$-failset $U^{s}$ proves necessity in (1). Since $U^{s}$ is an $\underline{\mathbf{M}}$-failset of $s$ within $\mathscr{R}$, we know that $\mathscr{P} \backslash U^{s}$ does not yield a duality on s. By the Retraction Test Algebra Lemma [20, Lemma 4], it follows that $\mathscr{R} \backslash U^{s}$ does not yield a duality on $\mathbf{r}$, for all $r \in U^{s}$. Thus condition (i) in (d) of Theorem 1.4 holds. To prove that $U^{s}$ is a globally minimal $\mathbf{M}$-failset within $\mathscr{R}$, it remains to prove (ii) in (d) of Theorem 1.4. But this follows immediately from the fact that, for any $r \in U^{s}$, the structure $\underset{\sim}{\mathbf{M}}=\langle M$; End( $(\underline{M}), r, \mathscr{T}\rangle$ yields a duality on $\mathcal{D}$ based on $\underline{M}$ as shown in (1). Hence (3) (i) holds. 
It remains to prove (3) (iii). Let $V$ be a globally minimal $\underline{\mathbf{M}}$-failset (within $\mathscr{R}$ ) disjoint from End $(\underline{M})$. Since, by (1), End $(\underline{M}) \cup\{r\}$ yields a duality on $\mathcal{D}$ for every $r \in U^{s}$, the failset $V$ must contain $r$ for every $r \in U^{s}$, whence $U^{s} \subseteq V$. As $U^{s}$ is an $\underline{\mathbf{M}}$-failset and $V$ is a globally minimal $\underline{\mathbf{M}}$-failset (within $\mathscr{R}$ ), we obtain $V=U^{s}$, as required.

Taking $\underline{\mathbf{M}}=\underline{\mathbf{D}}$ yields the following interesting corollary.

COROLlARY 3.4. Let $\underline{\mathrm{D}}$ be a finite almost endodualisable but not endodualisable algebra with $s \subseteq D^{2}$ as the extra relation and assume that the set $U^{s}$ is a failset of $s$ within $\mathscr{B}(\underline{D})$. Let $\mathcal{D}:=\mathbb{V} \mathbb{P}(\underline{D})$.

(1) For all $r \in \mathscr{B}(\mathbf{D})$, the structure $\mathbb{D}=\langle D ; \operatorname{End}(\underline{D}), r, \mathscr{T}\rangle$ yields a duality on $\mathbb{D}$ if and only if $r \in U^{s}$.

(2) Each $r \in U^{s}$ serves as a test algebra showing that $\underline{\mathbf{D}}$ is not endodualisable.

(3) $U^{s}$ is a globally minimal failset within $\mathscr{B}(\mathbf{D})$.

(4) $U^{s} \cap \operatorname{End}(\underline{D})=\varnothing$ and $U^{s}$ is the only globally minimal failset within $\mathscr{B}(\underline{D})$ which is disjoint from $\operatorname{End}(\underline{D})$.

\section{Transferring optimal dualities: practice}

We turn now from theory to practice and present several applications which illustrate the utility of the general theory.

Kleene algebras with fixpoints First we see how to transfer an optimal duality based on the three-element Kleene algebra to other Kleene algebras that have a threeelement subalgebra. The variety of Kleene algebras $\mathcal{K}=\mathbb{I S P}(\underline{\mathbf{K}})$ is generated by the algebra $\underline{\mathbf{K}}=\langle\{0, a, 1\} ; \vee, \wedge, \neg, 0,1\rangle$ with $0<a<1$ and $\neg 0=1, \neg 1=0$ and $\neg a=a$. For any Kleene algebra $\mathbf{A} \in \mathcal{K}$, one can define two important sublattices, namely a filter $A^{\vee}:=\{c \vee \neg c \mid c \in A\}$ and an ideal $A^{\wedge}:=\{c \wedge \neg c \mid c \in A\}$ which are anti-isomorphic via the mapping $c \mapsto \neg c$. If $\mathbf{A}$ is a subalgebra of $\underline{K}^{n}$, then $\rho_{j}:=\pi_{j} \Upsilon_{\mathbf{A}}: \mathbf{A} \rightarrow \underline{\mathbf{K}}$ denotes the restriction to $\mathbf{A}$ of the $j$ th projection $\pi_{j}: \underline{\mathbf{K}}^{n} \rightarrow \underline{\mathbf{K}}$. The next lemma is easily proved.

LEMMA 4.1. Let A be a Kleene algebra.

(i) A is a Boolean algebra if and only if $\left|A^{\vee}\right|=1$.

(ii) A has a subalgebra isomorphic to $\underline{\mathbf{K}}$ if and only if $A^{\vee} \cap A^{\wedge}=\{d\}$, where $d=\neg d$ is the unique $\neg$-fixpoint of $\mathbf{A}$.

(iii) $\mathbf{A}$ has the five-element Kleene algebra chain as a retract if and only if $\mathbf{A}$ has a subalgebra isomorphic to $\underline{\mathrm{K}}$ and $A^{\vee}$ is a non-Boolean lattice.

(iv) If $\mathbf{A}$ is a subalgebra of $\underline{\mathbf{K}}^{n}$, then $A^{\wedge}=A \cap\{0, a\}^{n}$ and $A^{\vee}=A \cap\{a, 1\}^{n}$. 
(v) If $\mathbf{A}$ is a subalgebra of $\underline{\mathbf{K}}^{n}$, then $\mathbf{A}$ has no subalgebra isomorphic to $\underline{\mathbf{K}}$ if and only if there is a projection $\rho_{i}: \mathbf{A} \rightarrow \underline{\mathbf{K}}$ satisfying $\rho_{i}(A)=\{0,1\}$.

Let $K_{0}:=\{0,1\}, \Delta_{K_{0}}:=\{(0,0),(1,1)\}$, let $\preceq$ be the order given by

$$
\preceq:=\{(0,0),(0, a),(a, a),(1, a),(1,1)\},
$$

let $\succeq$ be the converse of $\preceq$ and let - be the binary relation $K^{2} \backslash\{(0,1),(1,0)\}$. In [13] (see also [2, Section 8.4]) it is shown that the subsets of $\mathbb{S}(\underline{\mathbf{K}}) \cup \mathbb{S}\left(\underline{\mathbf{K}}^{2}\right)$ that yield optimal dualities on $\mathcal{K}$ based on $\underline{\mathbf{K}}$ are the transversals of the following globally minimal failsets:

- $\{-\}$,

- $\{\preceq, \geq\}$,

- $U_{K_{0}}:=\left\{K_{0}, \Delta_{K_{0}}, K_{0}^{2}, K \times K_{0}, K_{0} \times K, \succeq \cap\left(K \times K_{0}\right), \preceq \cap\left(K_{0} \times K\right)\right\}$.

The set $\operatorname{End}(\underline{\mathbf{K}})$ clearly plays no role here. Hence, for arbitrary $r \in U_{K_{0}}$,

$$
\underline{\mathbf{K}}_{1}=\langle\{0, a, 1\} ; r, \preceq,-, \mathscr{T}\rangle \quad \text { and } \quad \mathbf{K}_{2}=\{\{0, a, 1\} ; r, \succeq,-, \mathscr{T}\rangle
$$

yield optimal dualities on $\mathcal{K}$ based on $\underline{\mathbf{K}}$.

By applying the Optimal Duality Transfer Theorem 2.4 we shall transfer these optimal dualities on $\underline{\mathbf{K}}$ to optimal dualities on any Kleene algebra $\underline{\mathbf{M}}$ that has $\underline{\mathbf{K}}$ as a subalgebra, that is, (up to isomorphism) to any $\underline{\mathbf{M}}$ that has a $\neg$-fixpoint. To do this, it suffices to verify that $\{r, s,-\}^{\circ}$ avoids $\underline{\mathbf{M}}$ for every $r \in U_{K_{0}}$ and $s \in\{\preceq, \succeq\}$. We shall achieve this by applying Lemma 2.5 and Lemma 2.6: in the next three lemmas we show that the relations in the optimal dualities given by $\underset{\sim}{\mathbf{K}_{1}}$ and $\underset{\sim}{\mathbf{K}}$ satisfy the required 'minimality' conditions.

LEMMA 4.2. Let $\underline{\mathrm{M}}$ be a Kleene algebra that has $\underline{\mathrm{K}}$ as a subalgebra and let $r \in U_{K_{0}}$.

(i) The map $\gamma^{\prime}: \mathcal{K}(\mathbf{r}, \underline{\mathbf{K}}) \rightarrow K$, defined by $\gamma^{\prime}(x):=$ a for all $x \in \mathcal{K}(\mathbf{r}, \underline{\mathbf{K}})$, satisfies

$$
\begin{aligned}
\text { Fail } \underline{\mathbf{r}}\left(\gamma^{\prime}\right) & =\left\{r_{1} \in \mathscr{B}(\underline{\mathbf{K}}) \mid \mathbf{r}_{1} \text { has no subalgebra isomorphic to } \underline{\mathbf{K}}\right\} \\
& =\left\{r_{1} \in \mathscr{B}(\underline{\mathbf{K}}) \mid \mathbf{K}_{0} \text { is a retract of } \mathbf{r}_{1}\right\} .
\end{aligned}
$$

(ii) The relation $r$ avoids $\underline{\mathbf{M}}$ relative to $\{r, \preceq,-\}$ and $\{r, \succeq,-\}$.

PROOF. Let $r \in U_{K_{0}}$. Inspection of $U_{K_{0}}$ shows that $\mathbf{r}$ has no $\neg$-fixpoint and therefore no subalgebra isomorphic to $\underline{\mathbf{K}}$. Let $r_{1} \in \mathscr{B}(\underline{\mathbf{K}})$ be $n$-ary and assume that $\mathbf{r}_{1}$ has no subalgebra isomorphic to $\underline{K}$, that is, $(a, \ldots, a) \notin r_{1}$. By Lemma $4.1(\mathrm{v})$, there is a projection $\rho_{i}: \mathbf{r} \rightarrow \underline{\mathbf{K}}$ such that $\rho_{i}(r)=\{0,1\}$. Hence, for all $c \in r$, we have 
$\left(\rho_{i}(c), \ldots, \rho_{i}(c)\right) \in\{(0, \ldots, 0),(1, \ldots, 1)\} \subseteq r_{1}$, whence $\left(\rho_{i}, \ldots, \rho_{i}\right) \in r_{1}^{\mathcal{K}(\mathrm{r}, \underline{\mathbf{K}})}$. But, $\left(\gamma^{\prime}\left(\rho_{i}\right), \ldots, \gamma^{\prime}\left(\rho_{i}\right)\right)=(a, \ldots, a) \notin r_{1}$. Thus, $r_{1} \in$ Fail $\frac{\mathbf{K}}{\mathbf{K}}\left(\gamma^{\prime}\right)$. If $\mathbf{r}_{1}$ has a subalgebra isomorphic to $\underline{\mathbf{K}}$, then $(a, \ldots, a) \in r_{1}$ and consequently $\gamma^{\prime}$ preserves $r_{1}$. To complete the proof of (i), it remains to show that $\mathbf{r}_{1}$ has no subalgebra isomorphic to $\underline{\mathbf{K}}$ if and only if $\mathbf{K}_{0}$ is a retract of $\mathbf{r}_{1}$, but this is an almost immediate consequence of Lemma 4.1 (v). Since $\mathbf{r}$ has no subalgebra isomorphic to $\underline{\mathbf{K}}$, for all $r \in U_{K_{0}}$, while $\preceq, \succeq$, and - do have subalgebras isomorphic to $\underline{\mathbf{K}}$, it follows from (i) and Lemma 2.6 that $r$ avoids $\underline{\mathbf{M}}$ relative to $\{r, \preceq,-\}$ and $\{r, \succeq,-\}$. This proves (ii).

LEMMA 4.3. Let $s=\preceq$ and let $r \in U_{K_{0}}$.

(i) The map $\gamma^{\prime}: \mathcal{K}(\mathbf{s}, \underline{\mathbf{K}}) \rightarrow K$, defined by

$$
\gamma^{\prime}(x):= \begin{cases}1 & \text { if } x(1, a)=a \\ a & \text { if } x(1, a)=1\end{cases}
$$

satisfies Fail $\frac{\mathbf{K}}{\mathbf{s}}\left(\gamma^{\prime}\right)=\left\{s_{1} \in \mathscr{B}(\underline{\mathbf{K}}) \mid \mathbf{s}_{1}\right.$ has $\mathbf{s}$ as a retract $\}$.

(ii) The relation $\preceq$ is as small as possible relative to $\{r, \preceq,-\}$.

(iii) Let $\underline{\mathrm{M}}$ be a Kleene algebra that does not have $\mathbf{s}$ as a retract. Then $\preceq$ avoids $\underline{\mathrm{M}}$ relative to $\{r, \preceq,-\}$.

Proof. It is easily seen that each homomorphism $u: \mathbf{A} \rightarrow \mathbf{B}$ satisfies $u\left(A^{\vee}\right) \subseteq B^{\vee}$. Since $K^{\vee}=\{a, 1\}$, the map $\gamma^{\prime}$ is well defined. To simplify the notation, define $a^{*}=1$ and $1^{*}=a$, that is, ${ }^{*}$ is Boolean complementation in the two-element chain $\{a, 1\}$. Denote the pointwise extension of * to $\{a, 1\}^{n}$ by ${ }^{*}$ also. Hence, $\gamma^{\prime}(x)=x(1, a)^{*}$, for all $x \in \mathcal{K}(\mathbf{s}, \underline{\mathbf{K}})$. Let $s_{1}$ be an $n$-ary algebraic relation on $\underline{\mathbf{K}}$.

Assume that $\mathbf{s}$ is not a retract of $\mathbf{s}_{1}$. Since $\mathbf{s}$ is a five-element chain, $s_{1}^{\vee}$ is a Boolean lattice by Lemma 4.1. Let $\left(x_{1}, \ldots, x_{n}\right) \in s_{1}^{\mathcal{K}(\mathbf{s}, \underline{\underline{K}})}$. It follows that

$$
\bar{a}:=(a, \ldots, a)=\left(x_{1}(a, a), \ldots, x_{n}(a, a)\right) \in s_{1} .
$$

Hence, $s_{1}^{\vee}$ is a Boolean sublattice of $\{a, 1\}^{n}$. Thus, $\left(x_{1}(1, a), \ldots, x_{n}(1, a)\right) \in s_{1}^{\vee}$ implies that

$$
\begin{aligned}
\left(\gamma^{\prime}\left(x_{1}\right), \ldots, \gamma^{\prime}\left(x_{n}\right)\right) & =\left(x_{1}(1, a)^{*}, \ldots, x_{n}(1, a)^{*}\right) \\
& =\left(x_{1}(1, a), \ldots, x_{n}(1, a)\right)^{*} \in s_{1}^{\vee} \subseteq s_{1} .
\end{aligned}
$$

Hence, $\gamma^{\prime}$ preserves $s_{1}$.

Now assume that $\gamma^{\prime}$ preserves $s_{1}$. Since we wish to prove that $\mathbf{s}$ is a not a retract of $s_{1}$, we may assume without loss of generality that $s_{1}$ includes the diagonal $\{\overline{0}, \bar{a}, \overline{1}\}$ of $K^{n}$. In order to prove that $\mathbf{s}$ is not a retract of $s_{1}$ it suffices to show that $s_{1}^{v}$ is a Boolean lattice, that is, that $s_{1}^{\vee}$ is closed under ${ }^{*}$. Let $c \in s_{1}^{\vee}$. Then $v: \mathbf{s} \rightarrow \mathbf{s}_{1}$, 
defined by $v(1,1)=\overline{1}, v(1, a)=c, v(a, a)=\bar{a}, v(0, a)=\neg c$, and $v(0,0)=\overline{0}$, is an embedding of $\mathbf{s}$ into $\mathbf{s}_{1}$. Define $x_{i}:=\rho_{i}^{\mathbf{s}_{1}} \circ v$. Then $\left(x_{1}, \ldots, x_{n}\right) \in s_{1}^{\mathcal{X}(\mathbf{s}, \underline{\mathbf{K}})}$. Since $\gamma^{\prime}$ preserves $s_{1}$, we conclude that

$$
\begin{aligned}
c^{*}=v(1, a)^{*} & =\left(x_{1}(1, a), \ldots, x_{n}(1, a)\right)^{*} \\
& =\left(x_{1}(1, a)^{*}, \ldots, x_{n}(1, a)^{*}\right)=\left(\gamma^{\prime}\left(x_{1}\right), \ldots, \gamma^{\prime}\left(x_{n}\right)\right) \in s_{1},
\end{aligned}
$$

as required. Hence, (i) holds. Let $r \in U_{K_{0}}$. Since $\mathbf{s}=\preceq$ is a retract of neither $\mathbf{r}$ nor - , (ii) and (iii) follow at once by Lemma 2.5 .

LEMMA 4.4. Let $t=-$ and let $r \in U_{K_{0}}$.

(i) The map $\gamma^{\prime}: \mathcal{K}(\mathbf{t}, \underline{\mathbf{K}}) \rightarrow K$, defined by

$$
\gamma^{\prime}(x):= \begin{cases}1 & \text { if } x(1, a)=a \\ 0 & \text { if } x(1, a)=1,\end{cases}
$$

satisfies Fail $\frac{\mathbf{K}}{\mathbf{K}}\left(\gamma^{\prime}\right)=\left\{t_{1} \in \mathscr{B}(\underline{\mathbf{K}}) \mid \mathbf{t}_{1}\right.$ has $\mathbf{t}$ as a retract $\}$.

(ii) The relation - is as small as possible relative to $\{r, \preceq,-\}$.

(iii) Let $\underline{\mathrm{M}}$ be a Kleene algebra that does not have $\mathrm{t}$ as a retract. Then - avoids $\underline{\mathrm{M}}$ relative to $\{r, \preceq,-\}$.

PROOF. First note that $\mathcal{K}(\mathbf{t}, \underline{\mathbf{K}})=\left\{\rho_{1}^{\mathrm{t}}, \rho_{2}^{\mathrm{t}}\right\}$ and hence $\gamma^{\prime}(x)=0 \Leftrightarrow x=\rho_{1}^{\mathbf{t}}$ and $\gamma^{\prime}(x)=1 \Leftrightarrow x=\rho_{2}^{\mathrm{t}}$. Let $t_{1}$ be an $n$-ary algebraic relation on $\underline{\mathbf{K}}$. Assume that $\gamma^{\prime}$ does not preserve $t_{1}$. Hence, there exist $x_{1}, \ldots, x_{n} \in \mathcal{K}(\mathbf{t}, \underline{\mathbf{K}})$ such that $\left(x_{1}, \ldots, x_{n}\right) \in t_{1}^{\mathcal{K}(\mathbf{t}, \mathbf{K})}$ but $\left(\gamma^{\prime}\left(x_{1}\right), \ldots, \gamma^{\prime}\left(x_{n}\right)\right) \notin t_{1}$. Let $\mathbf{t}_{1}^{\prime}$ be the subalgebra of $\underline{\mathbf{K}}^{n}$ generated by $t_{1}$ and the $n$-tuple $\left(\gamma^{\prime}\left(x_{1}\right), \ldots, \gamma^{\prime}\left(x_{n}\right)\right)$. Suppose now that $(0,1) \in$ $\left(\pi_{i} \sqcap \pi_{j}\right)\left(t_{1}\right)$ for all $i \neq j$. Thus, $\{0,1\}^{2} \subseteq\left(\pi_{i} \sqcap \pi_{j}\right)\left(t_{1}\right)$. Since $\left(\pi_{i} \sqcap \pi_{j}\right)\left(\mathbf{t}_{1}^{\prime}\right)$ is the subalgebra of $\underline{\mathbf{K}}^{2}$ generated by $\left(\pi_{i} \sqcap \pi_{j}\right)\left(t_{1}\right)$ and the ordered pair $\left(\gamma^{\prime}\left(x_{i}\right), \gamma^{\prime}\left(x_{j}\right)\right)$ and since $\gamma^{\prime}\left(x_{i}\right), \gamma^{\prime}\left(x_{j}\right) \in\{0,1\}$, it follows that $\left(\pi_{i} \sqcap \pi_{j}\right)\left(t_{1}^{\prime}\right)$ and $\left(\pi_{i} \sqcap \pi_{j}\right)\left(t_{1}\right)$ are equal. Since every algebraic relation on a finite lattice-based algebra is determined by its binary projections (see Baker and Pixley [1]), we conclude that $t_{1}=t_{1}^{\prime}$ contradicting the fact that $\left(\gamma^{\prime}\left(x_{1}\right), \ldots, \gamma^{\prime}\left(x_{n}\right)\right) \in t_{1}^{\prime} \backslash t_{1}$. Thus, there exist $i$ and $j$ such that $\left(\gamma^{\prime}\left(x_{i}\right), \gamma^{\prime}\left(x_{j}\right)\right)=(0,1) \notin\left(\pi_{i} \sqcap \pi_{j}\right)\left(t_{1}\right)$. In particular, we have $x_{i}=\rho_{1}^{\mathrm{t}}$ and $x_{j}=\rho_{2}^{\mathrm{t}}$. It follows that $\left(\pi_{i} \sqcap \pi_{j}\right)\left(t_{1}\right) \subseteq K^{2} \backslash\{(0,1),(1,0)\}=t$. Thus, $u:=\rho_{i}^{\mathbf{t}_{1}} \sqcap \rho_{j}^{\mathbf{t}_{1}}: \mathbf{t}_{1} \rightarrow \mathbf{t}$ is well defined. Define $\nu: t \rightarrow t_{1}$ by $\nu:=x_{1} \sqcap \cdots \sqcap x_{n}$. Then, for all $\left(c_{1}, c_{2}\right) \in t$, we have

$$
\begin{aligned}
(u \circ v)\left(c_{1}, c_{2}\right) & =\left(\rho_{i}^{\mathfrak{t}_{1}} \sqcap \rho_{j}^{\mathbf{t}_{1}}\right)\left(x_{1}\left(c_{1}, c_{2}\right), \ldots, x_{n}\left(c_{1}, c_{2}\right)\right) \\
& =\left(x_{i}\left(c_{1}, c_{2}\right), x_{j}\left(c_{1}, c_{2}\right)\right)=\left(c_{1}, c_{2}\right) \text { as } x_{i}=\rho_{1}^{\mathbf{t}} \text { and } x_{j}=\rho_{2}^{\mathbf{t}} .
\end{aligned}
$$

Hence, $t$ is a retract of $t_{1}$. 
Conversely, assume that $t$ is a retract of $t_{1}$. Let $v: t \rightarrow t_{1}$ be the embedding and let $u: t_{1} \rightarrow \mathbf{t}$ be the corresponding retraction. Define $x_{i}:=\rho_{i}^{\mathbf{t}_{1}} \circ v$. Then $\left(x_{1}, \ldots, x_{n}\right) \in t_{1}^{\mathcal{X}(t, \underline{K})}$. Suppose that $\left(\gamma^{\prime}\left(x_{1}\right), \ldots, \gamma^{\prime}\left(x_{n}\right)\right) \in t_{1}$. Note that the $n$ tuple $\left(\gamma^{\prime}\left(x_{1}\right), \ldots, \gamma^{\prime}\left(x_{n}\right)\right)$ is a Boolean element of $t_{1}$, that is, $\left(\gamma^{\prime}\left(x_{1}\right), \ldots, \gamma^{\prime}\left(x_{n}\right)\right) \in$ $t_{1} \cap\{0,1\}^{n}$. Consequently, $u\left(\gamma^{\prime}\left(x_{1}\right), \ldots, \gamma^{\prime}\left(x_{n}\right)\right) \in\{(0,0),(1,1)\}$. Assume that $u\left(\gamma^{\prime}\left(x_{1}\right), \ldots, \gamma^{\prime}\left(x_{n}\right)\right)=(1,1)$. Recall that

Since

$$
\gamma^{\prime}\left(x_{i}\right)= \begin{cases}0 & \text { if } x_{i}=\rho_{1}^{\mathrm{t}} \\ 1 & \text { if } x_{i}=\rho_{2}^{\mathrm{t}}\end{cases}
$$

$$
v((a, 1))_{i}=\left(x_{1} \sqcap \cdots \sqcap x_{n}\right)((a, 1))_{i}= \begin{cases}a & \text { if } x_{i}=\rho_{1}^{\mathrm{t}} \\ 1 & \text { if } x_{i}=\rho_{2}^{\mathrm{t}},\end{cases}
$$

we have $\left(\gamma^{\prime}\left(x_{1}\right), \ldots, \gamma^{\prime}\left(x_{n}\right)\right) \leqslant v((a, 1))$. Hence,

$$
(1,1)=u\left(\gamma^{\prime}\left(x_{1}\right), \ldots, \gamma^{\prime}\left(x_{n}\right)\right) \leqslant u(v((a, 1)))=(a, 1),
$$

a contradiction. Now assume that $u\left(\gamma^{\prime}\left(x_{1}\right), \ldots, \gamma^{\prime}\left(x_{n}\right)\right)=(0,0)$. Since

$$
v((1, a))_{i}=\left(x_{1} \sqcap \cdots \sqcap x_{n}\right)((1, a))_{i}= \begin{cases}1 & \text { if } x_{i}=\rho_{1}^{\mathrm{t}} ; \\ a & \text { if } x_{i}=\rho_{2}^{\mathrm{t}},\end{cases}
$$

we have $\neg\left(\gamma^{\prime}\left(x_{1}\right), \ldots, \gamma^{\prime}\left(x_{n}\right)\right) \leqslant v((1, a))$. Hence,

$$
\begin{aligned}
(1,1)=\neg(0,0) & =\neg u\left(\gamma^{\prime}\left(x_{1}\right), \ldots, \gamma^{\prime}\left(x_{n}\right)\right) \\
& =u\left(\neg \gamma^{\prime}\left(x_{1}\right), \ldots, \neg \gamma^{\prime}\left(x_{n}\right)\right) \leqslant u(\nu((1, a)))=(1, a),
\end{aligned}
$$

a contradiction. Hence, $\left(\gamma^{\prime}\left(x_{1}\right), \ldots, \gamma^{\prime}\left(x_{n}\right)\right) \notin t_{1}$ and consequently $\gamma^{\prime}$ does not preserve $t_{1}$. This proves (i), and since $\mathbf{t}=-$ is a retract of neither $\preceq$ nor $\mathbf{r}$, for all $r \in U_{K_{0}}$, Lemma 2.5 yields (ii) and (iii).

We require one more elementary lemma.

LEMMA 4.5. (i) The algebra $\mathbf{K}_{0}$ is a retract of the 1-generated free Kleene algebra $\mathbf{F X}(1)$.

(ii) The algebras $\mathbf{s}=\preceq$ and $\mathbf{t}=-$ are not retracts of any finitely generated free Kleene algebra $\mathrm{FK}(n)(n \geqslant 1)$.

PROOF. As an ordered set, $\mathbf{F K}(1)$ is the linear $\operatorname{sum} 1 \oplus \mathbf{2}^{2} \oplus 1$, the doubly irreducible elements being the free generator $x$ and its negation $\neg x$. It is trivial that $K_{0}$ is a retract of $\mathbf{F K}(1)$. Hence (i) holds. Statement (ii) follows from the fact that, for all $n \geqslant 1$, 
the free Kleene algebra $\mathbf{F K}(n)$ has no subalgebra isomorphic to $\underline{\mathbf{K}}$ while both $\mathbf{s}$ and $\mathbf{t}$ do. This can also be seen easily via natural duals. The natural dual $\mathcal{K}(\mathrm{F} \mathcal{K}(n), \underline{\mathbf{K}})$ can be identified with $\underset{\sim}{\mathbf{K}}$ for any structure $\underset{\sim}{\mathbf{K}}$ algebraic over $\underline{\mathbf{K}}$ (see [2, page 45]), and so has a non-empty relation $K_{0}$. But because $\mathbf{s}$ and $\mathbf{t}$ have $\neg$-fixpoints, the relation $K_{0}$ is empty on both $\mathcal{K}(\mathbf{s}, \underline{\mathbf{K}})$ and $\mathcal{K}(\mathbf{t}, \underline{\mathbf{K}})$. Therefore, neither $\mathbf{s}$ nor $\mathbf{t}$ can be a retract of $\mathbf{F X}(n)$.

Our first conclusion does not concern the transfer of the duality given by $-\preceq$ and $K_{0}$ to some generating algebra other than $\underline{\mathbf{K}}$. Rather, it shows that this duality is optimally optimal in the whole class $\mathscr{B}(\underline{\mathbf{K}})$ in a very natural sense.

THEOREM 4.6. Let $R$ be a subset of $\mathscr{B}(\underline{\mathbf{K}})$ and let $s=\preceq$ and $t=-$. If the structure $\underset{\sim}{\mathbf{K}}:=\langle K ; R, \mathscr{T}\rangle$ yields a duality on $\mathcal{K}$ based on $\underline{\mathbf{K}}$, then there exist relations $r, s_{1}, t_{1} \in R$ such that $\mathbf{K}_{0}$, $\mathbf{s}$ and $\mathbf{t}$ are retracts of $\mathbf{r}, \mathbf{s}_{1}$ and $\mathbf{t}_{1}$, respectively.

PROOF. If $R$ yields a duality on $\mathcal{K}$ based on $\underline{\mathbf{K}}$, then $R$ must intersect every failset in $\mathscr{B}(\mathbf{K})$. The result follows at once from Lemma 4.2 (i), Lemma 4.3 (i) and Lemma 4.4 (i).

The main result of this subsection follows immediately from the above analysis by the Optimal Duality Transfer Theorem 2.4. The fact that $\underline{M}$ in (i) below is not 1endoprimal follows from Lemma 4.5 and the corollary of the Retraction Test Algebra Lemma [20, Corollary 5]. We note that if $\underline{\mathbf{M}}$ has $\underline{\mathbf{K}}$ as a subalgebra, then $\underline{\mathbf{M}}$ has a $\neg$-fixpoint, and consequently, for every relation $r \in U_{K_{0}}$, the corresponding algebra $\mathbf{r}$ is not a retract of $\underline{\mathbf{M}}$. Since $\underline{\mathbf{K}}$ is injective in $\mathcal{K}$, the algebra $\underline{\mathbf{M}}$ has $\underline{\mathbf{K}}$ as a subretract and consequently the unary relation $K$ on $\underline{\mathbf{M}}$ is not needed. We reiterate that, as $\operatorname{End}(\underline{K})=\left\{\mathrm{id}_{\underline{K}}\right\}$, the endomorphisms of $\underline{\mathbf{K}}$ play no role here.

THEOREM 4.7. Let $\underline{\mathrm{M}}$ be a finite Kleene algebra and assume that $\underline{\mathrm{M}}$ has $\underline{\mathbf{K}}$ as a subalgebra. Let $s=\preceq$ and $t=-$.

(i) Assume that $\underline{\mathbf{M}}$ has $\mathbf{t}$ and $\mathbf{s}$ as retracts. Then for any relation $r \in U_{K_{0}}$, the structure $\underline{\mathbf{M}}=\langle M ; \underline{\operatorname{End}}(\underline{\mathbf{M}}), r, \mathscr{T}\rangle$ yields a duality on $\mathcal{K}$ based on $\underline{\mathbf{M}}$ which is optimal modulo endomorphisms. Moreover, $\underline{\mathbf{M}}$ is not 1-endoprimal.

(ii) Assume that $\underline{\mathbf{M}}$ has $\mathbf{t}$ as a retract but does not have $\mathbf{s}$ as a retract. Then for any relation $r \in U_{K_{0}}$, the structure $\mathbf{M}=\langle M ; \operatorname{End}(\underline{\mathbf{M}}), s, r, \mathscr{T}\rangle$ yields a duality on $\mathcal{K}$ based on $\underline{\mathbf{M}}$ which is optimal modulo endomorphisms.

(iii) Assume that $\underline{\mathbf{M}}$ has $\mathbf{s}$ as a retract but does not have $\mathbf{t}$ as a retract. Then for any relation $r \in U_{K_{0}}$, the structure $\mathbf{M}=\langle M ; \operatorname{End}(\underline{\mathbf{M}}), t, r, \mathscr{T}\rangle$ yields a duality on $\mathcal{K}$ based on $\underline{\mathbf{M}}$ which is optimal modulo endomorphisms.

(iv) Assume that $\underline{\mathbf{M}}$ has neither $\mathbf{s}$ nor $\mathbf{t}$ as a retract. Then for any relation $r \in U_{\kappa_{0}}$, the structure $\mathbf{M}=\langle M ; \operatorname{End}(\underline{\mathbf{M}}), t, s, r, \mathscr{T}\rangle$ yields a duality on $\mathcal{K}$ based on $\underline{\mathbf{M}}$ which is optimal modulo endomorphisms. 
Semilattices The varieties of semilattices, bounded, upper-bounded and lowerbounded semilattices are $\mathcal{S}_{K}=\mathbb{\| S P}\left(\underline{2}_{K}\right)$ where, respectively, $\underline{\mathbf{2}}_{K}=\langle\{0,1\} ; \vee, K\rangle$ is the 2-element semilattice with bounds $K=\varnothing, K=\{0,1\}, K=\{1\}$ and $K=\{0\}$. Let

$$
\begin{aligned}
& \underset{\sim}{\mathbf{2}}:=\langle\{0,1\} ; \vee, 0,1, \mathscr{T}), \quad{\underset{\sim}{201}}_{2}:=\langle\{0,1\} ; \vee, \mathscr{T}\rangle, \\
& {\underset{\sim}{1}}_{1}:=\langle\{0,1\} ; \vee, 1, \mathscr{T}\rangle, \quad{\underset{\sim}{0}}_{0}:=\langle\{0,1\} \vee, 0, \mathscr{T}\rangle \text {, }
\end{aligned}
$$

where, as usual, $\mathscr{T}$ is the discrete topology. In Davey and Werner [14] it was shown that ${\underset{\sim}{\boldsymbol{2}} K}_{K}$ yields a duality on $\boldsymbol{S}_{K}$ (see also [10]). Hence, in each case, $\underline{\boldsymbol{2}}_{K}$ is almost endodualisable with graph $(V)$ as the extra relation which is, qua algebra, isomorphic to $\underline{\underline{2}}_{K}^{2}$.

One can prove that the set $V:=\left\{s_{1} \in \mathscr{B}\left(\underline{\mathbf{2}}_{K}\right) \mid \underline{\mathbf{2}}_{K}^{2}\right.$ is a retract of $\left.\mathbf{s}_{1}\right\}$ contains a failset of $s:=\operatorname{graph}(V) \subseteq\{0,1\}^{3}$ for each choice of $K$. We note that since $\underline{\mathbf{2}}_{K}$ is injective in $\mathcal{S}_{K}$, one can replace " $\underline{2}_{K}^{2}$ is a retract of $\mathbf{s}_{1}$ " by " $\mathbf{s}_{1}$ has a subalgebra isomorphic to $\underline{\mathbf{2}}_{K}^{2}$ ".

Our Almost Endodualisability Transfer Theorem 3.1 provides an alternative approach to the results on semilattices presented in [10]. Let $\underline{\mathbf{M}}$ be a non-trivial finite semilattice in $\boldsymbol{S}_{K}$. The Almost Endodualisability Transfer Theorem 3.1 gives us that $\underline{\mathbf{M}}$ is almost endodualisable with extra relation $s$. Using Theorem 3.1 and the fact that $V$ contains a failset of $s$, one can show that $\underline{\mathbf{M}}$ is endodualisable if and only if $\underline{\mathbf{M}}$ has a subalgebra isomorphic to $\underline{\underline{2}}_{K}^{2}$, and that if $\underline{\mathrm{M}}$ is not endodualisable, then $\underline{\mathbf{2}}_{K}^{2}$, and any $\mathbf{t} \in \mathcal{S}_{K}$ having a subalgebra isomorphic to $\underline{\mathbf{2}}_{K}^{2}$, serves as a test algebra showing that $\underline{\mathbf{M}}$ is not endodualisable.

The smallest $k$-generated free algebra $\mathrm{FS}_{K}(k)$ that has the algebra $\underline{2}_{K}^{2}$ as a retract exists in the varieties $\mathcal{S}_{K}$ where $K=\varnothing$ and $K=\{0\}$ with $k=3$ and $k=2$, respectively. Using Part (3) of Theorem 3.1 one can prove that if $K=\varnothing(K=\{0\})$, then $\underline{M}$ is endodualisable if and only if $\underline{M}$ is not a tree ( $\underline{M}$ is not a chain) if and only if $\underline{\mathbf{M}}$ is endoprimal if and only if $\underline{\mathbf{M}}$ is 3-endoprimal ( $\underline{\mathbf{M}}$ is 2-endoprimal).

It is known that in cases $K=\{1\}$ and $K=\{0,1\}$ there is no $k$-generated free algebra $\mathbf{F S}_{K}(k)$ that has the algebra $\underline{2}_{K}^{2}$ as a retract (see also [20]). In these varieties, examples of finite semilattices that are endoprimal but not endodualisable were found in [10].

Bounded distributive lattices The variety $\mathcal{D}$ of bounded distributive lattices is $\mathbb{U S P}(\underline{\mathbf{D}})$ where $\underline{\mathbf{D}}=\langle\{0,1\} ; \vee, \wedge, 0,1\rangle$ is the two-element bounded lattice. Priestley duality for $\mathcal{D}$ states, in part, that the structure $\underset{\sim}{\mathbf{D}}=\left\langle\{0,1\} ; \leqslant_{D}, \mathscr{T}\right\rangle$ yields a duality on $\mathcal{D}$, where $\leqslant_{D}=\{(0,0),(0,1),(1,1)\}$ is the usual order on $\{0,1\}$ (see [2]). Hence $\underline{D}$ is almost endodualisable with $\leqslant_{D}$ as the extra relation. To apply Theorem 3.3 , we require a characterisation of the algebraic relations $r \in \mathscr{B}:=\mathscr{B}(\underline{D})$ that have $\leqslant_{D}$ as a retractive projection. 
We prove the following lemma in a slightly more general setting than we need in this subsection as the more general version will be required in the following subsection. If $\left\{i_{1}, \ldots, i_{k}\right\}$ is a $k$-element subset of $\{1, \ldots, n\}$, then we denote the natural $\left\{i_{1}, \ldots, i_{k}\right\}$ projection by $\pi_{i_{1}, \ldots, i_{k}}: \underline{\mathbf{D}}^{n} \rightarrow \underline{\mathbf{D}}^{k}$. Let $\underline{\mathbf{2}}:=\langle\{0,1\} ; \vee, \wedge\rangle$ be the two-element lattice.

LEMMA 4.8. Let $r \in \mathscr{B}(2)$ with $\mathbf{r} \leqslant \underline{2}^{n}$. The following are equivalent:

(i) the lattice $\mathbf{r}$ is non-Boolean;

(ii) there exist $i, j \in\{1, \ldots, n\}$ with $i \neq j$ such that $\pi_{i, j}(\mathbf{r})$ is a non-Boolean sublattice of $\underline{\mathbf{2}}^{2}$, that is, $\pi_{i, j}(\mathbf{r})$ is a three-element chain;

(iii) $\leqslant_{D}$ is a retractive projection of $r$;

(iv) 3 is a retract of $\mathbf{r}$.

ProOF. The implication (iii) $\Rightarrow$ (iv) is trivial as $\leqslant_{D}$, qua lattice, is a three-element chain, while (iv) $\Rightarrow$ (i) is trivial since a homomorphic image of a Boolean lattice is Boolean.

We now prove the contrapositive of (i) $\Rightarrow$ (ii) via a 'Baker-Pixley-like' argument (see [1]). Assume that $\pi_{i, j}(\mathbf{r})$ is Boolean for all $i \neq j$. Denote the top and bottom of $\mathbf{r}$ by $T_{r}$ and $\perp_{r}$, respectively. Let $\left(a_{1}, \ldots, a_{n}\right) \in r$. We shall prove by induction that $r$ contains the relative complement $\left(a_{1}^{\prime}, \ldots, a_{n}^{\prime}\right)$ of $\left(a_{1}, \ldots, a_{n}\right)$ in the interval $\left[\perp_{r}, T_{r}\right]$ within $\underline{2}^{n}$.

Let $2 \leqslant k<n$ and assume that $\left(a_{i_{1}}^{\prime}, \ldots, a_{i_{k}}^{\prime}\right) \in \pi_{i_{1}, \ldots, i_{k}}(r)$ for all $k$-element subsets $\left\{i_{1}, \ldots, i_{k}\right\}$ of $\{1, \ldots, n\}$. Let $\left\{i_{0}, i_{1}, \ldots, i_{k}\right\}$ be a $(k+1)$-element subset of $\{1, \ldots, n\}$. By the inductive hypothesis, for each $j \in\left\{i_{0}, \ldots, i_{k}\right\}$ we have

$$
\left(a_{i_{0}}^{\prime}, \ldots, a_{i_{j-1}}^{\prime}, a_{i_{j+1}}^{\prime}, \ldots, a_{i_{k}}^{\prime}\right) \in \pi_{i_{0}, \ldots, i_{j-1}, i_{j+1}, \ldots, i_{k}}(r),
$$

and thus there is an element $b_{j} \in\{0,1\}$ such that

$$
\mathbf{b}_{j}:=\left(a_{i_{0}}^{\prime}, \ldots, a_{i_{j-1}}^{\prime}, b_{j}, a_{i_{j+1}}^{\prime}, \ldots, a_{i_{k}}^{\prime}\right) \in \pi_{i_{0}, \ldots, i_{k}}(r) .
$$

Define a $(k+1)$-ary term by $n\left(v_{0}, \ldots, v_{k}\right):=\left(v_{0} \wedge v_{1}\right) \vee\left(v_{1} \wedge v_{2}\right) \vee\left(v_{2} \wedge v_{0}\right)$. It is easy to check that $\boldsymbol{n}$ is a near-unanimity term on $\underline{2}$, that is, that $\underline{\mathbf{2}}$ satisfies

$$
n(u, v, v, \ldots, v) \approx n(v, u, v, \ldots, v) \approx \ldots \approx n(v, v, \ldots, v, u) \approx v
$$

Since $\pi_{i_{0}, \ldots, i_{k}}(r)$ is closed under $n$, we obtain

$$
\left(a_{i_{0}}^{\prime}, \ldots, a_{i_{k}}^{\prime}\right)=n\left(\mathbf{b}_{0}, \ldots, \mathbf{b}_{k}\right) \in \pi_{i_{0}, \ldots, i_{k}}(r),
$$

as required. Since $\pi_{i, j}(\mathbf{r})$ is Boolean for all $i \neq j$, by assumption, the inductive hypothesis is fulfilled for $k=2$. 
Finally we prove that (ii) $\Rightarrow$ (iii). Assume that $\pi_{i, j}(\mathbf{r})$ is non-Boolean. It follows that $\pi_{i, j}(r)=\leqslant_{D}$ or $\pi_{i, j}(r)=\geqslant_{D}$. Since $\pi_{i, j}(r)=\geqslant_{D}$ implies that $\pi_{j, i}(r)=\leqslant_{D}$, we may assume that $s:=\pi_{i, j}(r)=\leqslant_{D}$. Let $\mathbf{a}, \mathbf{b}, \mathbf{c} \in r$ be elements such that $\pi_{i, j}(\mathbf{a})=(0,0)$, $\pi_{i, j}(\mathbf{b})=(0,1)$ and $\pi_{i, j}(\mathbf{c})=(1,1)$. Let $s:=\leqslant_{D}$; then the embedding $q: \mathbf{s} \rightarrow \mathbf{r}$ defined by $q((0,0))=\mathbf{a}, q((0,1))=\mathbf{a} \vee \mathbf{b}$ and $q((1,1))=\mathbf{a} \vee \mathbf{b} \vee \mathbf{c}$ satisfies $\pi_{i, j} \circ q=\mathrm{id}_{\mathrm{s}}$, whence $\leqslant_{D}$ is a retractive projection of $r$.

As a lattice, $\leqslant_{D}$ is a three-element chain. Consequently, the three-element chain, $\mathbf{3}=\langle\{0, a, 1\} ; \vee, \wedge, 0,1\}$, is a test algebra showing that $\underline{\mathbf{D}}$ is not endodualisable. This is witnessed by the map $\gamma^{\prime}: \mathcal{D}(3, \underline{\mathbf{D}}) \rightarrow D$ mapping each $x \in \mathcal{D}(\mathbf{3}, \underline{\mathrm{D}})$ to the complement $x(a)^{\prime}$ of the element $x(a)$ in $D=\{0,1\}$. As was first noted in [7], the map $\gamma^{\prime}$ preserves a relation $r$ in $\mathscr{B}(\mathbf{D})$ if and only if $\mathbf{r}$ is Boolean. The reader can easily see, that the equivalence of (i) and (iii) in Lemma 4.8 also holds in the category of bounded distributive lattices. Hence

$$
\text { Fail } \begin{aligned}
\frac{\mathbf{D}}{3}\left(\gamma^{\prime}\right) & =\left\{r \in \mathscr{B} \mid \leqslant_{D} \text { is a retractive projection of } r\right\} \\
& =\{r \in \mathscr{B} \mid \mathbf{r} \text { is non-Boolean }\} .
\end{aligned}
$$

It is obvious that for any non-trivial finite bounded distributive lattice $\underline{M}$ we can regard $\underline{\mathbf{D}}$ as a subretract of $\underline{\mathbf{M}}$. The following theorem, which provides alternative proofs and refinements of results proved in [7], is now an immediate consequence of Theorem 3.3.

THEOREM 4.9. Let $U:=\{r \in \mathscr{B}(\underline{\mathrm{D}}) \mid \mathbf{r}$ is non-Boolean $\}$ and let $\underline{\mathbf{M}}$ be a finite Boolean lattice.

(1) The algebra $\underline{\mathbf{M}}$ is almost endodualisable: let $r \in \mathscr{B}(\underline{\mathbf{D}})$, then

$$
\mathbf{M}=\langle M ; \operatorname{End}(\underline{\mathbf{M}}), r, \mathscr{T}\rangle
$$

yields a duality on $\mathcal{D}$ based on $\underline{\mathrm{M}}$ if and only if $r \in U$.

(2) For every $r \in U$, the algebra $\mathbf{r}$ serves as a test algebra showing that $\underline{\mathbf{M}}$ is not endodualisable.

(3) Let $\mathscr{R}:=\operatorname{End}(\underline{\mathbf{M}}) \cup \mathscr{B}(\underline{\mathrm{D}})$. Then

(i) $U$ is a globally minimal $\underline{\mathbf{M}}$-failset within $\mathscr{R}$,

(ii) $U \cap$ End $(\underline{M})=\varnothing$,

(iii) $U$ is the only globally minimal $\underline{\mathbf{M}}$-failset within $\mathscr{R}$ that is disjoint from $\operatorname{End}(\underline{\mathbf{M}})$.

Finally, as $F \mathcal{D}(1) \cong \mathbf{3}$ is the smallest free algebra in $\mathcal{D}$ that has 3 as a retract, it follows from the Almost Endodualisability Transfer Theorem 3.1 that a finite nontrivial bounded distributive lattice $\underline{\mathbf{M}}$ is endoprimal if and only if $\underline{\mathbf{M}}$ is 1-endoprimal if and only if $\underline{M}$ is endodualisable if and only if 3 is a retract of $\underline{M}$ if and only if $\underline{\mathbf{M}}$ is non-Boolean, which was first proved in [7]. It is straightforward to modify the arguments given here to obtain the corresponding results in [7] and [5] for the two-element lattice with only one or with no bounds as nullary operations. 
Stone algebras The variety of Stone algebras is $S t=\mathbb{Q S P}(\underline{\mathbf{S}})$ where the generator $\underline{\mathbf{S}}=\left\langle\{0, a, 1\} ; \vee, \wedge,{ }^{*}, 0,1\right)$ is the 3-element chain with $0^{*}=1$ and $a^{\star}=1^{*}=0$. The structure $\mathbf{S}=\langle\{0, a, 1\} ; e, \preccurlyeq, \mathscr{T}\rangle$, where $e \in \operatorname{End}(\underline{\mathbf{S}})$ is determined by $e(a):=1$ and $\preccurlyeq:=\{(0,0),(a, a),(1,1),(1, a)\}$, yields a duality on $S t$ (see Davey [3] or [2, page 105]). Hence $\underline{\mathbf{S}}$ is almost endodualisable with $\preccurlyeq$ as the extra relation. In order to apply Theorem 3.3, we require a characterisation of the algebraic relations $r \in \mathscr{B}(\underline{S})$ that have $\preccurlyeq$ as a retractive projection.

Since the four-element chain, $\mathbf{4}=\left\{\{0, b, c, 1\} ; \vee, \wedge,{ }^{*}, 0,1\right)$, where $0<b<c<$ 1 , is isomorphic to $\preccurlyeq$ (regarded as a subalgebra of $\underline{S}^{2}$ ), we can use $\mathbf{4}$ as our test algebra for showing that $\underline{\mathbf{S}}$ is not endodualisable. As each $x \in \mathcal{S} t(\mathbf{4}, \underline{\mathbf{S}})$ maps the dense filter $d(\mathbf{4})=\{b, c, 1\}$ to the (Boolean) dense filter $d(\mathbf{S})=\{a, 1\}$, we may define a function $\gamma^{\prime}: S t(4, S) \rightarrow S$ by mapping each $x \in \mathcal{S} t(4, \underline{S})$ to the relative complement of $x(c)$ in the interval $[x(b), 1] \subseteq\{a, 1\}$.

LEMMA 4.10. Let $\gamma^{\prime}: \mathcal{S} t(4, \underline{S}) \rightarrow S$ be defined as above and let $r \in \mathscr{B}$. The following are equivalent:

(i) $d(\mathbf{r})$ is a non-Boolean lattice;

(ii) $\preccurlyeq$ is a retractive projection of $r$;

(iii) 4 is a retract of $\mathbf{r}$;

(iv) $r \in$ Fail $\frac{\mathbf{s}}{4}\left(\gamma^{\prime}\right)$.

PROof. Let $r \in \mathscr{B}$ and assume that $r$ is $n$-ary. The equivalence of (i) and (iii) is well known (see, for example, [5]). If $r$ has $\preccurlyeq$ as a retractive projection, then $r$ entails $\preccurlyeq$. Since it is clear that $\gamma^{\prime}$ does not preserve $\preccurlyeq$, it follows that $\gamma^{\prime}$ does not preserve $r$. Hence, (ii) implies (iv). We now prove the contrapositive of (iv) $\Rightarrow$ (i). Assume that $d(\mathbf{r})$ is Boolean and let $x_{1}, \ldots, x_{n} \in \mathcal{S} t(\mathbf{4}, \underline{\mathbf{S}})$ with $\left(x_{1}, \ldots, x_{n}\right) \in r^{\mathcal{S}(t, \mathbb{S})}$. Then $\left(\gamma^{\prime}\left(x_{1}\right), \ldots, \gamma^{\prime}\left(x_{n}\right)\right)$ is the relative complement $\boldsymbol{d}$ of $\left(x_{1}(c), \ldots, x_{n}(c)\right)$ in the interval $\left[\left(x_{1}(b), \ldots, x_{n}(b)\right),(1, \ldots, 1)\right] \subseteq\{a, 1\}^{n}$. Since $\left(x_{1}(b), \ldots, x_{n}(b)\right),\left(x_{1}(c), \ldots, x_{n}(c)\right) \in r$ and since $\mathbf{r}$ is Boolean, we have $d \in r$, that is, $\left(\gamma^{\prime}\left(x_{1}\right), \ldots, \gamma^{\prime}\left(x_{n}\right)\right) \in r$. Hence, $\gamma^{\prime}$ preserves $r$. Thus (iv) implies (i). It remains to prove that (i) implies (ii). Assume that $d(\mathbf{r})$ is non-Boolean. Since $d(\mathbf{r})$ is a sublattice of $d(\underline{\mathbf{S}})^{n} \cong \underline{\mathbf{2}}^{n}$, we can apply Lemma 4.8 . Hence there exist $i, j$ such that $\pi_{i, j}(d(\mathbf{r}))$ is a non-Boolean sublattice of $\{a, 1\}^{2}$. Without loss of generality, we may assume that $\pi_{i, j}(d(r))=\{(a, a),(1, a),(1,1)\}$. It follows that $\pi_{i, j}(r)=\preccurlyeq$, as required.

We can now apply Theorem 3.3 to obtain the following result. Note that if $\underline{M}$ is a non-Boolean Stone algebra, then $\underline{\mathbf{M}}$ has a subalgebra isomorphic to $\underline{\mathbf{S}}$ and there is no loss of generality in assuming that $\underline{\mathbf{S}}$ is actually a subalgebra of $\underline{\mathbf{M}}$. Since $\underline{\mathbf{S}}$ is injective in $\boldsymbol{S} t$, it follows that $\underline{\mathbf{S}}$ is subretract of $\underline{\mathbf{M}}$. 
THEOREM 4.11. Let $\underline{\mathbf{M}}$ be a finite non-Boolean Stone algebra such that $d(\underline{\mathrm{M}})$ is Boolean and assume that $\underline{\mathbf{S}}$ is a subalgebra of $\underline{\mathbf{M}}$. Let $U:=\{r \in \mathscr{B}(\underline{\mathbf{S}}) \mid d(\mathbf{r})$ is non-Boolean\}.

(1) The algebra $\underline{\mathbf{M}}$ is almost endodualisable: let $r \in \mathscr{B}(\underline{\mathbf{S}})$, then

$$
\underset{\sim}{\mathbf{M}}=\langle M ; \operatorname{End}(\underline{\mathbf{M}}), r, \mathscr{T}\rangle
$$

yields a duality on $\mathbf{S} t$ based on $\underline{\mathbf{M}}$ if and only if $r \in U$.

(2) For every $r \in U$, the algebra $\mathbf{r}$ serves as a test algebra showing that $\underline{\mathbf{M}}$ is not endodualisable.

(3) Let $\mathscr{R}:=\operatorname{End}(\underline{\mathbf{M}}) \cup \mathscr{B}(\underline{\mathbf{S}})$. Then

(i) $U$ is a globally minimal $\underline{\mathbf{M}}$-failset within $\mathscr{R}$,

(ii) $U \cap \operatorname{End}(\underline{M})=\varnothing$,

(iii) $U$ is the only globally minimal $\underline{\mathbf{M}}$-failset within $\mathscr{R}$ that is disjoint from $\operatorname{End}(\underline{\mathbf{M}})$.

The smallest free algebra in $\mathcal{S} t$ having 4 as a retract is $\mathbf{F S} t$ (2) (see Grätzer [18, page 188], where free Stone algebras are described). The only Stone algebras that do not have $\underline{\boldsymbol{S}}$ as a retract are the Boolean algebras (and these are endodualisable). Therefore it follows from the Almost Endodualisability Transfer Theorem 3.1 that a finite non-Boolean Stone algebra $\underline{\mathbf{M}}$ is endoprimal if and only if $\underline{\mathbf{M}}$ is 2-endoprimal if and only if $\underline{\mathbf{M}}$ is endodualisable if and only if $\underline{\mathbf{M}}$ has $\mathbf{4}$ as a retract if and only if the dense filter $d(\underline{M})$ is a non-Boolean lattice, which was first proved in [5].

\section{Proofs of the technical lemmas}

Our main transfer theorems, namely Theorem 2.4, Theorem 3.1 and Theorem 3.3, were quite easy to prove given Lemma 2.2 and Lemma 2.3. We now present the proofs of these technical lemmas. Their proofs depend upon four even more technical lemmas!

Let $\underline{\mathbf{M}}$ be finite algebra in $\mathcal{D}=\mathbb{N S P}(\underline{\mathbf{D}})$ that has $\underline{\mathbf{D}}$ as a subalgebra. As in the assumptions for the Subalgebra Duality Transfer Theorem, let $\Omega=\left\{\omega_{1}, \ldots, \omega_{k}\right\}$ be a set of endomorphisms of $\underline{\mathbf{M}}$ which satisfy

(E1) $\omega_{i}(M) \subseteq D$ for all $i$, and

(E2) $\omega_{1}, \ldots, \omega_{k}$ separate the points of $M$,

and let $\omega:=\omega_{1} \sqcap \cdots \sqcap \omega_{k}: \underline{\mathbf{M}} \rightarrow \underline{\mathbf{D}}^{k}$ be the induced embedding. Assume that $\underline{\mathbf{D}}=\langle D ; \operatorname{End}(\underline{\mathbf{D}}), S, \mathscr{T}\rangle$ yields an optimal duality on $\mathcal{D}$ modulo endomorphisms and let $s \in S$. Then $s$ is needed in $R:=\operatorname{End}(\underline{D}) \cup S$ and so there exists a map $\gamma^{\prime}: \mathcal{D}(\mathbf{s}, \underline{\mathbf{D}}) \rightarrow D$ satisfying (N1) and (N2). As we aim to investigate the optimality of the transferred duality for $\mathcal{D}$ based on $\underline{\mathrm{M}}$, we must use the map $\gamma^{\prime}$ to define a map 
$\gamma: \mathcal{D}(\mathbf{s}, \underline{\mathbf{M}}) \rightarrow M$ whose failset Fail $\frac{\mathbf{M}}{\mathbf{s}}(\gamma)$ can be used to study entailment amongst the relations occurring in the transferred duality.

Assume that $s$ is $n$-ary so that $\mathbf{s} \leqslant \underline{\mathbf{D}}^{n}$. Let $\left.\omega^{-1}: \omega \underline{\mathbf{M}}\right) \rightarrow \underline{\mathbf{M}}$ be the inverse of the embedding $\omega: \underline{\mathbf{M}} \rightarrow \underline{\mathbf{D}}^{k}$ and 'define' the map $\gamma: \mathcal{D}(\mathbf{s}, \underline{\mathbf{M}}) \rightarrow M$ by

$$
\gamma(x):=\omega^{-1}\left(\gamma^{\prime}\left(\omega_{1} \circ x\right), \ldots, \gamma^{\prime}\left(\omega_{k} \circ x\right)\right),
$$

for each $x \in \mathcal{D}(\mathbf{s}, \underline{\mathbf{M}})$. Note that

$$
\omega_{i}(\gamma(x))=\gamma^{\prime}\left(\omega_{i} \circ x\right)
$$

The following four lemmas will establish sufficient conditions for $\gamma$ to be both well defined and well behaved.

LEMMA 5.1. If $\gamma^{\prime}$ preserves the $k$-ary algebraic relation $\omega(M) \subseteq D^{k}$, and in particular if $\gamma^{\prime}$ preserves every $k$-ary algebraic relation $r$ on $\underline{\mathbf{D}}$ such that $\mathbf{r}$ is isomorphic to $\underline{\mathbf{M}}$, then $\gamma: \mathcal{D}(\mathbf{s}, \underline{\mathbf{M}}) \rightarrow M$ is well defined.

Proof. Assume that $\gamma^{\prime}$ preserves the relation $\omega(M)$ and let $x \in \mathcal{D}(\mathbf{s}, \underline{\mathbf{M}})$. Since

$$
\left(\omega_{1} \circ x, \ldots, \omega_{k} \circ x\right) \in \omega(M)^{\mathcal{D}(s, \mathbf{D})},
$$

we conclude that $\left(\gamma^{\prime}\left(\omega_{1} \circ x\right), \ldots, \gamma^{\prime}\left(\omega_{k} \circ x\right)\right) \in \omega(M)$, whence

$$
\gamma(x):=\omega^{-1}\left(\left(\gamma^{\prime}\left(\omega_{1} \circ x\right), \ldots, \gamma^{\prime}\left(\omega_{k} \circ x\right)\right)\right) \in M,
$$

as required.

LEMMA 5.2. If $e \in \operatorname{End}(\underline{\mathrm{M}})$ and $\gamma^{\prime}$ preserves the $k$-ary algebraic partial operations on $\underline{\mathbf{D}}$ defined by

$$
\left.e_{i}:=\omega_{i} \circ e \circ \omega^{-1}: \omega \underline{\mathbf{M}}\right) \rightarrow \underline{\mathbf{D}},
$$

for $i=1, \ldots, k$, then $\gamma$ is well defined and preserves $e$. Consequently, if $\gamma^{\prime}$ preserves every $(k+1)$-ary algebraic relation $r$ on $\underline{\mathbf{D}}$ such that $\mathbf{r}$ is isomorphic to $\underline{\mathbf{M}}$, then $\gamma$ is well defined and preserves $\operatorname{End}(\underline{\mathbf{M}})$.

PROOF. Let $e \in \operatorname{End}(\underline{\mathbf{M}})$ and assume that $\gamma^{\prime}$ preserves $e_{i}$ for $i=1, \ldots, k$. It follows that $\gamma^{\prime}$ preserves $\operatorname{dom}\left(e_{i}\right)=\omega(M)$, whence $\gamma$ is well defined. To show that $\gamma(e \circ x)=e(\gamma(x))$, for every $x \in \mathcal{D}(\mathbf{s}, \underline{\mathbf{M}})$, it suffices to prove that, for all $i=1, \ldots, k$,

$$
\omega_{i}(\gamma(e \circ x))=\omega_{i}(e(\gamma(x)))
$$


since the maps $\omega_{1}, \ldots, \omega_{k}: \underline{\mathbf{M}} \rightarrow \underline{\mathbf{D}}$ separate the points of $M$. Let $x \in D(\mathbf{s}, \underline{\mathbf{M}})$ and for all $i=1, \ldots, k$, define $y_{i}: s \rightarrow D$ by

$$
y_{i}:=\omega_{i} \circ x
$$

Then

$$
\omega^{-1} \circ\left(y_{1} \sqcap \cdots \sqcap y_{k}\right)=x .
$$

Now let $i \in\{1, \ldots, k\}$. By (5.3), we have

$$
\left(y_{1}, \ldots, y_{k}\right) \in \omega(M)^{\mathcal{D}(\mathbf{s}, \underline{D})}=\operatorname{dom}\left(e_{i}\right)^{\mathcal{D}(\mathbf{s}, \mathbf{D})} .
$$

Since $\gamma^{\prime}$ preserves the partial operation $e_{i}$, we have

$$
\gamma^{\prime}\left(e_{i}\left(y_{1}, \ldots, y_{k}\right)\right)=e_{i}\left(\gamma^{\prime}\left(y_{1}\right), \ldots, \gamma^{\prime}\left(y_{k}\right)\right) .
$$

Thus,

$$
\begin{aligned}
\left(\omega_{i} \circ e\right)(\gamma(x)) & =\left(\omega_{i} \circ e \circ \omega^{-1}\right)\left(\gamma^{\prime}\left(y_{1}\right), \ldots, \gamma^{\prime}\left(y_{k}\right)\right) & & \text { by (5.1) and (5.6) } \\
& =e_{i}\left(\gamma^{\prime}\left(y_{1}\right), \ldots, \gamma^{\prime}\left(y_{k}\right)\right) & & \text { by (5.4) } \\
& =\gamma^{\prime}\left(e_{i}\left(y_{1}, \ldots, y_{k}\right)\right) & & \text { by (5.8) } \\
& =\gamma^{\prime}\left(e_{i} \circ\left(y_{1} \sqcap \cdots \sqcap y_{k}\right)\right) & & \\
& =\gamma^{\prime}\left(\omega_{i} \circ e \circ \omega^{-1} \circ\left(y_{1} \sqcap \cdots \sqcap y_{k}\right)\right) & & \text { by (5.4) } \\
& =\gamma^{\prime}\left(\omega_{i} \circ(e \circ x)\right) & & \text { by (5.7) } \\
& =\omega_{i}(\gamma(e \circ x)) & & \text { by (5.2) }
\end{aligned}
$$

and hence (5.5) holds. Consequently, $\gamma$ preserves $e$. Since $\gamma^{\prime}$ preserves the $k$-ary partial operation $e_{i}$ if and only if it preserves the $(k+1)$-ary relation graph $\left(e_{i}\right)$, which as an algebra is isomorphic to $\left.\operatorname{dom}\left(e_{i}\right)=\omega \underline{\mathbf{M}}\right)$ and hence to $\underline{\mathbf{M}}$, the final claim follows immediately.

Let $r$ be an $m$-ary algebraic relation on $\underline{\mathbf{D}}$. By using the embedding $\omega \Gamma_{D}: \underline{\mathbf{D}} \rightarrow \underline{\mathbf{D}}^{k}$, we can assign a $\mathrm{km}$-ary algebraic relation $r_{\omega}$ on $\underline{\mathrm{D}}$ to the relation $r$ via

$$
r_{\omega}:=\left\{\left(\omega_{1}\left(c_{1}\right), \ldots, \omega_{k}\left(c_{1}\right), \ldots, \omega_{1}\left(c_{m}\right), \ldots, \omega_{k}\left(c_{m}\right)\right) \mid\left(c_{1}, \ldots, c_{m}\right) \in r\right\} .
$$

Note that, for any $\left(d_{11}, \ldots, d_{1 k}\right), \ldots,\left(d_{m 1}, \ldots, d_{m k}\right) \in \omega(M) \subseteq D^{k}$, we have:

$$
\begin{aligned}
& \left(d_{11}, \ldots, d_{1 k}, \ldots, d_{m 1}, \ldots, d_{m k}\right) \in r_{\omega} \\
\Longleftrightarrow & \left(\omega^{-1}\left(d_{11}, \ldots, d_{1 k}\right), \ldots, \omega^{-1}\left(d_{m 1}, \ldots, d_{m k}\right)\right) \in r .
\end{aligned}
$$

We shall use relations of the form $r_{\omega}$ in the proofs of the following two lemmas. 
LEMMA 5.3. Assume that $\gamma^{\prime}$ preserves the $k$-ary relation $\omega(M)$ and, for each $i$, preserves the endomorphism $\left.\omega_{i}\right|_{D} \in \operatorname{End}(\underline{D})$. If $\gamma^{\prime}$ preserves a finitary algebraic relation $r$ on $\underline{\mathrm{D}}$ then $\gamma$ also preserves $r$. In particular, $\gamma$ preserves the unary relation $D \subseteq M$.

PROOF. Let $r$ be $m$-ary. To show that $\gamma$ preserves $r$, let $\left(x_{1}, \ldots, x_{m}\right) \in r^{\mathcal{D}(s, \underline{M})}$. Then, for all $a \in s$, we have $\left(x_{1}(a), \ldots, x_{m}(a)\right) \in r \subseteq D^{m}$, whence $x_{i}(s) \subseteq D$ and $\left(x_{1}, \ldots, x_{m}\right) \in r^{\mathcal{D}(\mathbf{s}, \mathbf{D})}$. As $\gamma^{\prime}$ preserves $r$, we have $\left(\gamma^{\prime}\left(x_{1}\right), \ldots, \gamma^{\prime}\left(x_{m}\right) \in r\right.$. Thus we find

$$
\left.\left(\omega_{1} \Gamma_{D}\left(\gamma^{\prime}\left(x_{1}\right)\right), \ldots, \omega_{k} \Gamma_{D}\left(\gamma^{\prime}\left(x_{1}\right)\right), \ldots, \omega_{1} \uparrow_{D}\left(\gamma^{\prime}\left(x_{m}\right)\right), \ldots, \omega_{k}\right\rceil_{D}\left(\gamma^{\prime}\left(x_{m}\right)\right)\right) \in r_{\omega}
$$

by the definition of $r_{\omega}$. As $\gamma^{\prime}$ preserves each $\omega_{i} \uparrow_{D} \in \operatorname{End}(\underline{\mathbf{D}})$, we obtain

$$
\left(\gamma^{\prime}\left(\omega_{1} \circ x_{1}\right), \ldots, \gamma^{\prime}\left(\omega_{k} \circ x_{1}\right), \ldots, \gamma^{\prime}\left(\omega_{1} \circ x_{m}\right), \ldots, \gamma^{\prime}\left(\omega_{k} \circ x_{m}\right)\right) \in r_{\omega}
$$

By (5.9), this means that

$$
\left(\omega^{-1}\left(\gamma^{\prime}\left(\omega_{1} \circ x_{1}\right), \ldots, \gamma^{\prime}\left(\omega_{k} \circ x_{1}\right)\right), \ldots, \omega^{-1}\left(\gamma^{\prime}\left(\omega_{1} \circ x_{m}\right), \ldots, \gamma^{\prime}\left(\omega_{k} \circ x_{m}\right)\right)\right) \in r,
$$

whence $\left(\gamma\left(x_{1}\right), \ldots, \gamma\left(x_{m}\right)\right) \in r$ by $(5.1)$.

LEMMA 5.4. Assume that $\gamma^{\prime}$ preserves the $k$-ary relation $\omega(M)$ and, for each $i$, preserves the endomorphism $\left.\omega_{i}\right|_{D} \in \operatorname{End}(\underline{D})$. If $\gamma^{\prime}$ does not preserve $s$, then neither does $\gamma$.

Proof. Assume that $\gamma^{\prime}$ does not preserve $s$. Thus there exist $x_{1}, \ldots, x_{n} \in \mathcal{D}(\mathbf{s}, \underline{\mathbf{D}})$ with $\left(x_{1}, \ldots, x_{n}\right) \in s^{\mathcal{D}(s, \underline{D})}$ and $\left(\gamma^{\prime}\left(x_{1}\right), \ldots, \gamma^{\prime}\left(x_{n}\right)\right) \notin s$. Since $x_{i} \in \mathcal{D}(\mathbf{s}, \underline{\mathbf{M}})$, for all $i$, it suffices to prove that $\left(\gamma\left(x_{1}\right), \ldots, \gamma\left(x_{n}\right)\right) \notin s$. Since $\left(\gamma^{\prime}\left(x_{1}\right), \ldots, \gamma^{\prime}\left(x_{n}\right)\right) \notin s$, we have

$$
\left(\omega_{1}\left(\gamma^{\prime}\left(x_{1}\right)\right), \ldots, \omega_{k}\left(\gamma^{\prime}\left(x_{1}\right)\right), \ldots, \omega_{1}\left(\gamma^{\prime}\left(x_{n}\right)\right), \ldots, \omega_{k}\left(\gamma^{\prime}\left(x_{n}\right)\right)\right) \notin s_{\omega}
$$

by the definition of $s_{\omega}$. As $\gamma^{\prime}\left(x_{i}\right) \in D$, for all $i$, and since $\gamma^{\prime}$ preserves all $\left.\omega_{i}\right|_{D} \in$ End(D), we obtain

$$
\left(\gamma^{\prime}\left(\omega_{1} \circ x_{1}\right), \ldots, \gamma^{\prime}\left(\omega_{k} \circ x_{1}\right), \ldots, \gamma^{\prime}\left(\omega_{1} \circ x_{n}\right), \ldots, \gamma^{\prime}\left(\omega_{k} \circ x_{n}\right)\right) \notin s_{\omega} .
$$

By (5.9) we can rewrite this as

$$
\left(\omega^{-1}\left(\gamma^{\prime}\left(\omega_{1} \circ x_{1}\right), \ldots, \gamma^{\prime}\left(\omega_{k} \circ x_{1}\right)\right), \ldots, \omega^{-1}\left(\gamma^{\prime}\left(\omega_{1} \circ x_{n}\right), \ldots, \gamma^{\prime}\left(\omega_{k} \circ x_{n}\right)\right)\right) \notin s,
$$

whence, by (5.1), we obtain the desired conclusion $\left(\gamma\left(x_{1}\right), \ldots, \gamma\left(x_{n}\right)\right) \notin s$. 
With these four lemmas in hand we can now prove Lemma 2.2 and Lemma 2.3.

PROOF OF LEMMA 2.2. Let $U=$ Fail $\frac{\mathbf{D}}{s}\left(\gamma^{\prime}\right)$ where $\gamma^{\prime}: \mathcal{D}(\mathbf{s}, \underline{\mathbf{D}}) \rightarrow D$ and assume that $\mathbf{t}$ is a retract of $\mathbf{r}$ for all $r \in U$. Since $\mathbf{t}$ is not a retract of $\underline{\mathbf{M}}$, the map $\gamma^{\prime}$ preserves every relation $r \in \mathscr{B}(\underline{\mathbf{D}})$ with $\mathbf{r} \cong \underline{\mathbf{M}}$, whence $\gamma: \mathcal{D}(\mathbf{s}, \underline{\mathbf{M}}) \rightarrow M$ is well defined and preserves End(M), by Lemma 5.2. Thus $V:=$ Fail $\underline{\mathbf{M}}(\gamma)$ contains no (graphs of) endomorphisms of $\underline{\mathbf{M}}$, whence (i) holds. As $\mathbf{t}$ is not a retract of $\underline{\mathbf{D}}$, the map $\gamma^{\prime}$ preserves End(D) and hence, by Lemma 5.3 and Lemma 5.4, (ii) holds. Since $s \in U$, it follows from (ii) that $s \in V$, and consequently $V$ is a failset of $s$.

Proof of LEMma 2.3. Assume that $s \in S$ avoids $\underline{M}$ relative to End(D) $\cup S$. Then, by definition, there is a map $\gamma^{\prime}: \mathcal{D}(\mathbf{s}, \underline{\mathbf{D}}) \rightarrow D$ which preserves every endomorphism of $\underline{\mathbf{D}}$, every relation in $S \backslash\{s\}$ and every relation $r$ satisfying $\mathbf{r} \cong \underline{\mathbf{M}}$ and does not preserve $s$. By Lemmas 5.1, 5.2, 5.3 and 5.4, the map $\gamma: \mathcal{D}(\mathbf{s}, \underline{\mathbf{M}}) \rightarrow M$ (is well defined and) preserves the endomorphisms of $\underline{\mathbf{M}}$, the relation $D$ and every relation in $S \backslash\{s\}$ but does not preserve $s$, whence $s$ is needed in $\operatorname{End}(\underline{M}) \cup S \cup\{D\}$. Thus (1) implies (2). If $s$ were a retract of $\underline{\mathbf{M}}$, then $s$ would be entailed by End( $\underline{\mathbf{M}})$, and hence $s$ would not be needed in End $(\underline{M}) \cup S \cup\{D\}$. Hence, (2) implies (3).

\section{References}

[1] K. A. Baker and A. F. Pixley, 'Polynomial interpolation and the Chinese remainder theorem for algebraic systems', Math. Z. 143 (1975), 165-174.

[2] D. M. Clark and B. A. Davey, Natural dualities for the working algebraist (Cambridge University Press, Cambridge, 1998).

[3] B. A. Davey, 'Dualities for Stone algebras, double Stone algebras, and relative Stone algebras', Colloq. Math. 46 (1982), 1-14.

[4] — 'Duality theory on ten dollars a day', in: Algebras and orders (eds. I. G. Rosenberg and G. Sabidussi), NATO Advanced Study Institute Series, Series C, Vol. 389 (Kluwer Academic Publishers, Dordrecht, 1993) pp. 71-111.

[5] _ - 'Dualisability in general and endodualisability in particular', in: Logic and algebra (Pontignano, 1994) (eds. A. Ursini and P. Aglianò), Lecture Notes in Pure and Appl. Math. 180 (Dekker, New York, 1996) pp. 437-455.

[6] B. A. Davey and M. Haviar, 'A schizophrenic operation which aids the efficient transfer of strong dualities', Houston J. Math 26 (2000), 215-222.

[7] B. A. Davey, M. Haviar and H. A. Priestley, 'Endoprimal distributive lattices are endodualisable', Algebra Universalis 34 (1995), 444-453.

[8] _ 'The syntax and semantics of entailment in duality theory', J. Symbolic Logic 60 (1995), $1087-1114$.

[9] —-, 'Kleene algebras: a case-study of clones and dualities from endomorphisms', Acta Sci. Math. (Szeged) 67 (2001), 325-351.

[10] B. A. Davey and J. G. Pitkethly, 'Endoprimal algebras', Algebra Universalis 38 (1997), 266-288. 
[11] B. A. Davey and H. A. Priestley, 'Optimal natural dualities', Trans. Amer. Math. Soc. 338 (1993), 655-677.

[12] - 'Optimal natural dualities for varieties of Heyting algebras', Studia Logica 56 (1996), 67-96.

[13] — , 'Optimal natural dualities II: general theory', Trans. Amer. Math. Soc. 348 (1996), 36733711.

[14] B. A. Davey and H. Werner, 'Dualities and equivalences for varieties of algebras', in: Contributions to lattice theory (Szeged, 1980) (eds. A. P. Huhn and E. T. Schmidt), Colloq. Math. Soc. János Bolyai 33 (North-Holland, Amsterdam, 1983) pp. 101-275.

[15] —_, 'Piggyback-dualitäten', Bull. Austral. Math. Soc. 32 (1985), 1-32.

[16] —-, 'Piggyback dualities', in: Lectures in universal algebra (eds. L. Szabó and A. Szendrei), Coll. Math. Soc. János Bolyai 43 (North-Holland, Amsterdam, 1986) pp. 61-83.

[17] B. A. Davey and R. Willard, 'The dualisability of a quasi-variety is independent of the generating algebra', Algebra Universalis 45 (2001), 103-106.

[18] G. Grätzer, Lattice theory. First concepts and distributive lattices (Freeman, San Francisco, 1971).

[19] - General lattice theory, 2nd Edition (Birkhaüser, Basel, 1998).

[20] M. Haviar and H. A. Priestley, 'A criterion for a finite endoprimal algebra to be endodualisable', Algebra Universalis 42 (1999), 183-193.

[21] — , 'Endodualisable and endoprimal finite double Stone algebras', Algebra Universalis 42 (1999), 107-130.

[22] M. Saramago, A study of natural dualities, including an analysis of the structure of failsets (Ph.D. Thesis, University of Lisbon, 1998).

[23] _ - 'Some remarks on dualisability and endodualisability', Algebra Universalis 43 (2000), $197-212$.

[24] M. J. Saramago and H. A. Priestley, 'Optimal natural dualities: the structure of failsets', Internat. J. Algebra Comput. 12 (2002), 407-436.

[25] C. B. Wegener, Natural dualities for varieties generated by lattice-structured algebras (Ph.D. Thesis, University of Oxford, 1999).

School of Mathematics

La Trobe University

VIC 3086

Australia

e-mail: B.Davey@latrobe.edu.au
Department of Mathematics

M. Bel University PdF, Ruzova 13

97401 Banska Bystrica Slovak Republic e-mail: haviar@pdf.umb.sk 